Nonlinear Processes in Geophysics, 12, 643-660, 2005

SRef-ID: $1607-7946 / \mathrm{npg} / 2005-12-643$

European Geosciences Union

(C) 2005 Author(s). This work is licensed

under a Creative Commons License.

\title{
Hamiltonian formulation of nonlinear travelling Whistler waves
}

\author{
G. M. Webb ${ }^{1}$, J. F. McKenzie ${ }^{1,2,3}$, E. M. Dubinin ${ }^{4}$, and K. Sauer ${ }^{4}$ \\ ${ }^{1}$ Institute of Geophysics and Planetary Physics, University of California Riverside, Riverside CA 92521, USA \\ ${ }^{2}$ School of Physics and School of Mathematical and Statistical Sci., Univ. of KwaZulu-Natal, Durban, 4041, South Africa \\ ${ }^{3}$ University of KwaZulu-Natal (Howard College), Durban, 4041, South Africa \\ ${ }^{4}$ Max-Planck-Institute für Sonnensystemforschung, Katlenburg-Lindau, Germany
}

Received: 12 January 2005 - Revised: 13 April 2005 - Accepted: 14 April 2005 - Published: 22 June 2005

Part of Special Issue "Nonlinear plasma waves-solitons, periodic waves and oscillations"

\begin{abstract}
A Hamiltonian formulation of nonlinear, parallel propagating, travelling whistler waves is developed. The complete system of equations reduces to two coupled differential equations for the transverse electron speed $u$ and a phase variable $\phi=\phi_{p}-\phi_{e}$ representing the difference in the phases of the transverse complex velocities of the protons and the electrons. Two integrals of the equations are obtained. The Hamiltonian integral $H$, is used to classify the trajectories in the $(\phi, w)$ phase plane, where $\phi$ and $w=u^{2}$ are the canonical coordinates. Periodic, oscilliton solitary wave and compacton solutions are obtained, depending on the value of the Hamiltonian integral $H$ and the Alfvén Mach number $M$ of the travelling wave. The second integral of the equations of motion gives the position $x$ in the travelling wave frame as an elliptic integral. The dependence of the spatial period, $L$, of the compacton and periodic solutions on the Hamiltonian integral $H$ and the Alfvén Mach number $M$ is given in terms of complete elliptic integrals of the first and second kind. A solitary wave solution, with an embedded rotational discontinuity is obtained in which the transverse Reynolds stresses of the electrons are balanced by equal and opposite transverse stresses due to the protons. The individual electron and proton phase variables $\phi_{e}$ and $\phi_{p}$ are determined in terms of $\phi$ and $w$. An alternative Hamiltonian formulation in which $\tilde{\phi}=\phi_{p}+\phi_{e}$ is the new independent variable replacing $x$ is used to write the travelling wave solutions parametrically in terms of $\tilde{\phi}$.
\end{abstract}

\section{Introduction}

Recently (Sauer et al., 2002; Dubinin et al., 2003; McKenzie et al., 2004) it has been shown that nonlinear, travelling whistler waves, can propagate at speeds in excess of the maximum, linear whistler speed in the form of a stationary structure in the travelling wave frame, within which is embedded

Correspondence to: G. M. Webb

(gmwebb@ucr.edu) finer spatial oscillations and has been called an oscilliton for that reason. The linear dispersion relation yields the necessary condition for the formation of an oscilliton which arises from the fact that the phase and the group speeds are equal at a frequency of one half the electron gyrofrequency where the phase speed is maximized. The analysis is carried out in the travelling wave frame in which the protons must be placed on an equal footing with the electrons since their Reynolds stresses are of the same order. In fact the protons and electrons exchange momentum through the mediating Maxwell magnetic stresses.

In the non-relativistic case, in which charge neutrality is an appropriate assumption, Dubinin et al. (2003) showed that for the case where the background magnetic field and the travelling wave velocity are parallel, that the system could be reduced to the following two coupled differential equations:

$$
\begin{aligned}
\frac{d u_{e}}{d x} & =M_{A}^{2} u_{e} \sin \phi, \\
\frac{d \phi}{d x} & =2 M_{A}^{2}(1+\cos \phi)-\left(1-u_{e}^{2} / \mu\right)^{-1 / 2},
\end{aligned}
$$

where $u_{e}$ is the magnitude of the transverse electron velocity. The transverse electron and proton velocities have been written in the complex, amplitude and phase form as: $u_{j \pm}=u_{j y} \pm i u_{j z}=\left|u_{j}\right| \exp \left( \pm i \phi_{j}\right)$ where $j=e, p$ refer to the electron and proton species. The phase $\phi=\phi_{p}-\phi_{e}$ denotes the difference of the phases for the protons and the electrons. The above equations pertain to the case of a parallel magnetic field configuration in which the constant background magnetic field $\mathbf{B}_{0}=B_{0} \mathbf{e}_{x}$ and the travelling wave velocity $\mathbf{U}$ are along the $\mathrm{x}$-axis. In Eqs. (1.1)-(1.2)

$$
\begin{aligned}
& x=\frac{X}{\ell_{\bar{m}}}, \quad \ell_{\bar{m}}=\frac{U}{\Omega_{\bar{m}}}, \\
& \Omega_{\bar{m}}=\frac{e B_{0}}{\bar{m}}, \quad \bar{m}=\frac{m_{p} m_{e}}{m_{p}+m_{e}}, \quad \mu=\frac{m_{p}}{m_{e}},
\end{aligned}
$$

where $X$ is the $x$-coordinate in the travelling wave frame, $m_{p}$ and $m_{e}$ are the proton and electron masses, and $\Omega_{\bar{m}}$ is the 
a)

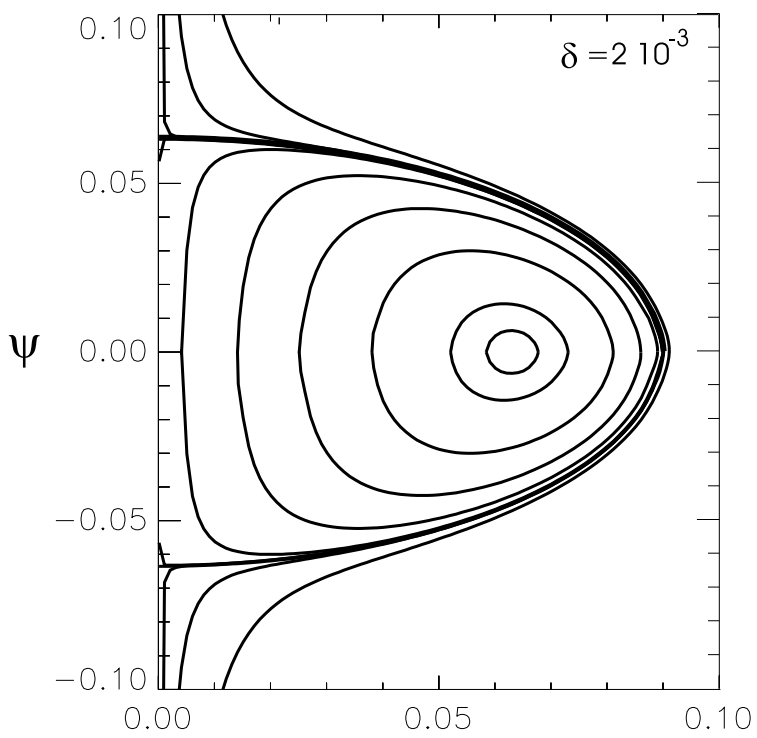

$\mathrm{u}$

b)

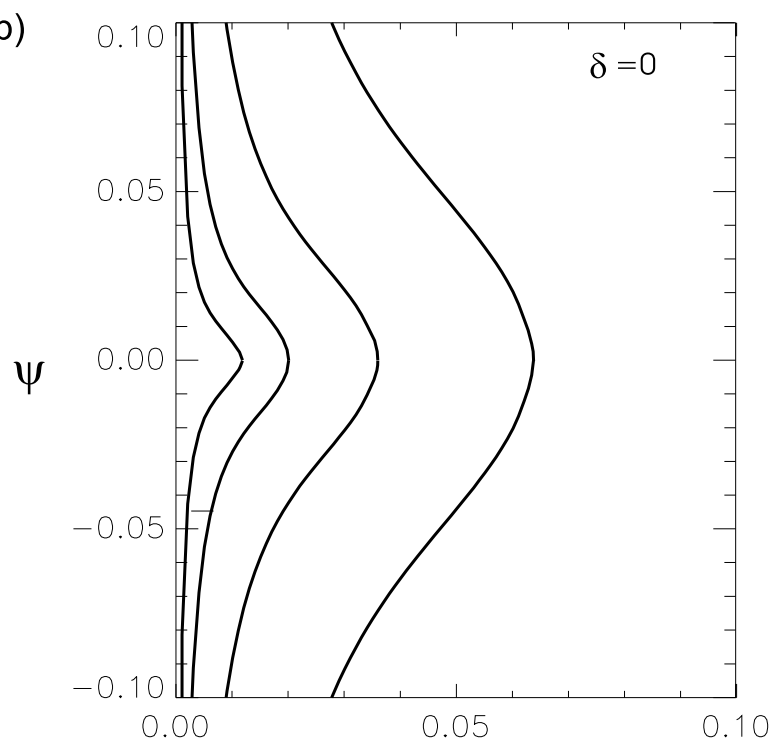

u

Fig. 1. Phase portraits of the system (Eq. 1.5) in the $(u, \psi)$ phase plane for: (a) $\delta=2 \times 10^{-3}$ and (b) $\delta=0$.

gyrofrequency corresponding to the geometric mean of the proton and electron masses $\bar{m}$. Since $m_{p} / m_{e}=1836$, then $\bar{m} \approx m_{e}$ to a good approximation. The equations:

$M_{A}^{2}=\frac{U^{2}}{V_{A \bar{m}}^{2}}, \quad V_{A \bar{m}}^{2}=\frac{B_{0}^{2}}{\mu_{0} n_{p} \bar{m}}$,

define the Alfvén Mach number $M_{A}$, where $V_{A \bar{m}}$ is the Alfvén speed based on the geometric mean $\bar{m}$ of the proton and electron masses. The normalizations used in Eqs. (1.1)(1.2) are slightly different than those used by Dubinin et al. (2003) (see Appendix A for the form of the equations using the Dubinin et al., 2003 normalizations).

Using the complex electron momentum equation for $u_{e \pm}=u_{e y} \pm i u_{e z}$ (Dubinin et al., 2003) we obtain the equation:

$\frac{d \phi_{e}}{d x}=\frac{\mu}{(\mu+1)\left(1-u_{e}^{2} / \mu\right)^{1 / 2}}-M_{A}^{2}(1+\cos \phi)$,

for $d \phi_{e} / d x$ throughout the wave. Combining Eqs. (1.2) and (1.5) for $\phi$ and $\phi_{e}$ we obtain the equation:

$\frac{d \phi_{p}}{d x}=M_{A}^{2}(1+\cos \phi)-\frac{1}{(\mu+1)\left(1-u_{e}^{2} / \mu\right)^{1 / 2}}$,

for $d \phi_{p} / d x$. Similarly,

$\frac{d \tilde{\phi}}{d x}=\frac{\mu-1}{(\mu+1)\left(1-u_{e}^{2} / \mu\right)^{1 / 2}}, \quad \tilde{\phi}=\phi_{p}+\phi_{e}$,

gives the variation of $d\left(\phi_{p}+\phi_{e}\right) / d x$ in the wave.

The assumption of charge neutrality $\left(n_{e}=n_{p}=n\right)$ implies $u_{p x}=u_{e x}=u_{x}$ for the $x$-components of the fluid velocities of the protons and the electrons. The total momentum and energy equations for the system yield the equations:

$u_{x}=\left(1-u_{e}^{2} / \mu\right)^{1 / 2}, \quad u_{p}=\frac{u_{e}}{\mu}$.

Equations (1.8) show that there is a limitation on the value of $u_{x}$ due to energy conservation constraints (the maximum value of $u_{x}$ is obtained when $u_{e}=0$ ), and that the transverse proton speed $u_{p}<<u_{e}$. The normalized, complex, transverse magnetic fields $B_{ \pm}=B_{y} \pm i B_{z}$ in the wave are given by the equations:

$B_{ \pm}=M_{A e}^{2}\left(\mu u_{p \pm}+u_{e \pm}\right)$,

where $M_{A e}=U / V_{A e}$ is the Alfvén Mach number based on the electron Alfvén speed $V_{A e}=B_{0} /\left(\mu_{0} n_{e} m_{e}\right)^{1 / 2}$.

The basic Eqs. (1.1)-(1.2) form an independent subsystem of the complete set of Eqs. (1.1)-(1.9). We show that the system (Eqs. 1.1-1.2) is in fact a Hamiltonian system in its own right. In this formulation, Eqs. (1.5)-(1.9) for $\phi_{e}, \phi_{p}, u_{x}$ and $B_{ \pm}$appear as auxiliary equations describing the interaction of the electron and proton fluids and magnetic fields in the wave. Dubinin et al. (2003) obtained the phase portrait integral:

$u_{e}^{2}(1+\cos \phi)+\frac{\mu}{M_{A}^{2}}\left(1-u_{e}^{2} / \mu\right)^{1 / 2}=\Phi=$ const.

Thus, in the $\left(\phi, u_{e}\right)$ phase plane, there is a family of periodic solutions around an $O$-point limited by a heteroclinic trajectory connecting two saddle points, which corresponds to the oscilliton solution (see Figs. 1 and 2).

Consider the phase portraits of the system (Eqs. 1.1-1.2) for the case where the Alfvén Mach number $M_{A e}=(1+\delta) / 2$, where $\delta$ is a small parameter (Dubinin et al., 2003). For small $\delta<<1$, the equation system has saddle critical points at $\left(u_{e}, \phi\right)=(0, \pm \sqrt{8 \delta})$ and has a centre critical point at 
a)
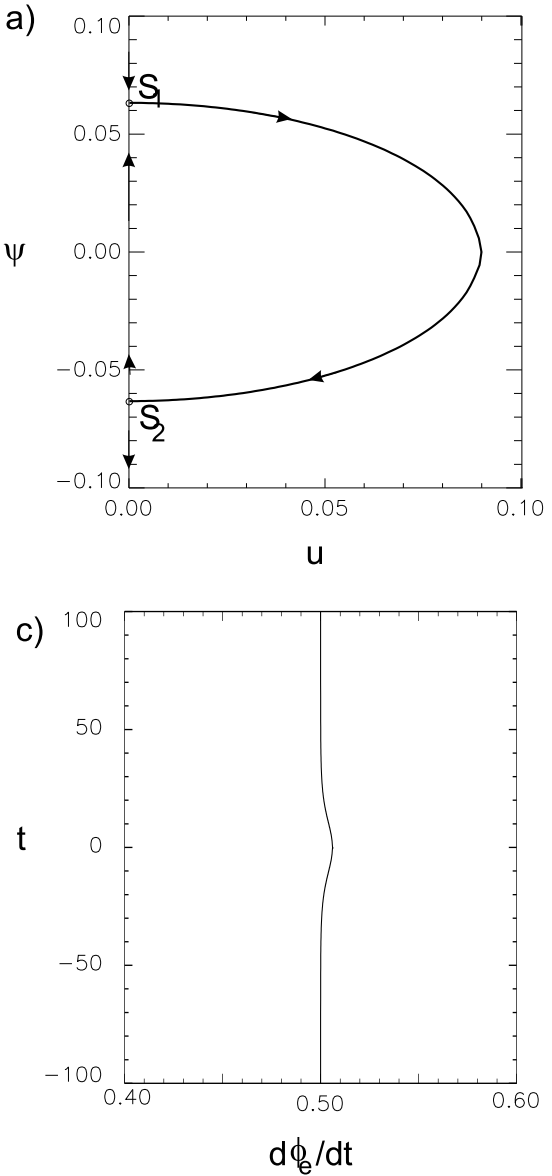

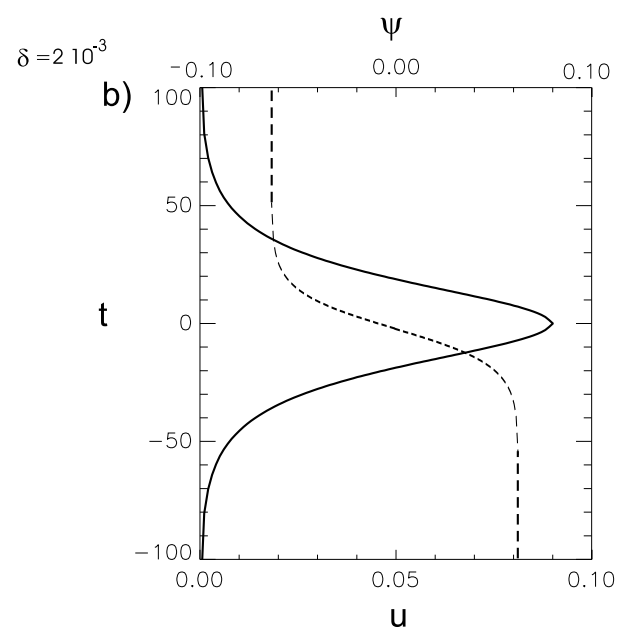

d)

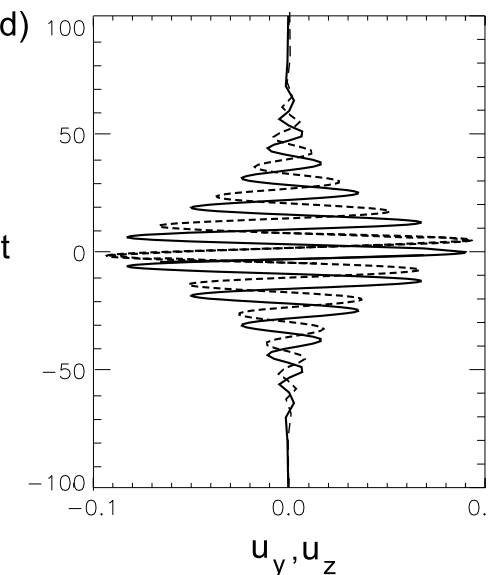

Fig. 2. Features of the oscilliton solution for the case $\delta=2 \times 10^{-3}$. Panel (a) shows the heteroclinic phase plane trajectory connecting the two saddle points; (b) shows the soliton like profile for $u$ (solid curve) and $\psi$ (dashed curve) obtained by integrating (Eq. 1.5); (c) shows the rate of change of the electron phase $d \phi_{e} / d t$ with normalized distance $t$ through the wave, and (d) shows fine scale oscillations of the electron transverse velocity components $u_{y}$ and $u_{z}$ within the large scale envelope for $u$ ( $u_{y}$ is the solid curve and $u_{z}$ is the dashed curve).

$\left(u_{e}, \phi\right)=(\sqrt{2 \mu \delta}, 0)$. The equation system possesses aproximate solutions for which $u_{e}^{2}<<\mu$ and $|\phi|<<1$. Using the new variables:

$\psi=M_{A e} \phi, \quad u=\frac{u_{e}}{\sqrt{2 \mu}}, \quad t=x M_{A e}$,

in Eqs. (1.1)-(1.2) yields the approximate equation system:

$\frac{d u}{d t}=\psi, \quad \frac{d \psi}{d t}=2 \delta-\psi^{2}-u^{2}$.

The detailed analysis of the approximate equation system is given in Dubinin et al. (2003). Figure 1 illustrates the phase portraits of the system (Eq. 1.12) in the $(u, \psi)$ phase plane for the case $\delta=2 \times 10^{-3}$ (upper panel) and for $\delta=0$ (lower panel). In the case $\delta=2 \times 10^{-3}$, the centre critical point at $(u, \psi)=(\sqrt{\delta}, 0)$ and the saddle critical points at $(u, \psi)=\left(0, \pm \sqrt{8 \delta} M_{A e}\right)$ are evident. As $\delta \rightarrow 0$, the centre and saddle critical points merge to $(0,0)$ (lower panel of Fig. 1).

Figure 2 illustrates the character of the oscilliton solution for the case $\delta=2 \times 10^{-3}$. The oscilliton solution corresponds to the heteroclinic orbit connecting the two saddle points $S_{1}$ and $S_{2}$ (Panel a). Figure $2 \mathrm{~b}$ illustrates the solution profiles for $u$ (the solid curve) and $\psi$ (the dashed curve) obtained by integrating Eq. (1.12). Panel (c) illustrates $d \phi_{e} / d t$, the rate of change of the electron phase $\phi_{e}$ with the normalized space variable $t$. Panel (d) shows the fine scale oscillations of the electron transverse velocity components $u_{y}$ and $u_{z}$, within the large scale envelope for $u$ ( $u_{y}$ is the solid curve and $u_{z}$ is the dashed curve). The name oscilliton comes from the fact that although the envelope of the wave for $u$ has the usual, single humped profile characteristic of a soliton, there are also fine scale oscillations of the transverse electron fluid velocity within the envelope.

The main aim of the paper is to show that the exact system (Eqs. 1.1-1.2) is a Hamiltonian system, that has two integrals, and hence is completely integrable without the need for any approximations. The first integral is the phase plane integral (Eq. 1.10) and is equivalent to the Hamiltonian of the system. There is a further exact integral for the system, which gives $x$ as a function of $u$ as an elliptic integral (Sect. 2). The fine scale variations of $\phi_{e}$ and 
$\phi_{p}$ throughout the wave envelope and the corresponding changes in the transverse complex velocities $u_{e \pm}$ and $u_{p \pm}$ and complex transverse magnetic field variations $B_{ \pm}$, are controlled by Eqs. (1.5)-(1.6). We determine the Hamiltonian critical points of Eqs. (1.1)-(1.2) (Sect. 2.1) and investigate the change in the phase portraits of the system with the Alfvén Mach number $M_{A}$ and the Hamiltonian integral $H$ (Sect. 2.2). The oscilliton (heteroclinic orbit) solution, which has an infinite spatial period is studied in Sect. 3. The dependence of the spatial period of the compacton and periodic wave solutions on $M_{A}$ and $H$ is determined in Sect. 4 . An alternative action-angle formulation of the Hamiltonian system is described in Appendix B. Appendix C describes a Hamiltonian formulation, in which $(\phi, \Lambda)$ are the canonical coordinates, $\tilde{\phi}=\phi_{e}+\phi_{p}$ is the new independent variable and $\Lambda=\left(1-u_{e}^{2} / \mu\right)^{1 / 2}$. In this formulation the travelling wave solutions are given parametrically in terms of $\tilde{\phi}$. The transformation simplifies the algebraic form of the solutions. Section 5 concludes with a summary and discussion.

\section{Hamiltonian formulation}

The system of Eqs. (1.1)-(1.2) can be written in the Hamiltonian form:

$$
\begin{aligned}
& \frac{d \phi}{d x}=\frac{\partial H_{0}}{\partial w} \equiv 2 M^{2}(1+\cos \phi)-(1-w / \mu)^{-1 / 2}, \\
& \frac{d w}{d x}=-\frac{\partial H_{0}}{\partial \phi} \equiv 2 M^{2} w \sin \phi,
\end{aligned}
$$

where

$$
\begin{aligned}
w & =u^{2}, \\
H_{0} & =2 M^{2} w(1+\cos \phi)+2 \mu(1-w / \mu)^{1 / 2} \equiv 2 M^{2} \Phi .
\end{aligned}
$$

In Eqs. (2.1)-(2.4) $M \equiv M_{A}$ is the Alfvén Mach number (Eq. 1.4); $u \equiv u_{e}$ is the transverse fluid speed for the electrons; and $\Phi$ is the phase space integral (Eq. 1.10) of Eqs. (1.1) and (1.2). Here, $(\phi, w) \equiv(q, p)$ are the canonical coordinates and $H_{0}=2 M^{2} \Phi$ is the Hamiltonian. Note that $H_{0}$ is a rescaled version of the phase space integral $\Phi$ in Eq. (1.10) which is a constant of the motion.

The Lagrangian $L$ and action $A$ for the system are given by the standard Legendre transformation:

$A=\int L d x$ where $L=w \phi_{x}-H_{0}$.

The Euler-Lagrange equations:

$$
\begin{aligned}
& \frac{\delta A}{\delta \phi}=2 M^{2} w \sin \phi-w_{x}=0, \\
& \frac{\delta A}{\delta w}=\phi_{x}-\left[2 M^{2}(1+\cos \phi)-(1-w / \mu)^{-1 / 2}\right]=0,
\end{aligned}
$$

are equivalent to the the equations of motion (Eqs. 1.1-1.2) or Eqs. (2.1)-(2.2).

The question now arises of whether it is possible to obtain a second integral or constant of the motion. To this end, we introduce the variables:

$$
\Lambda=(1-w / \mu)^{1 / 2}, \quad \tau=\tan (\phi / 2) \text {. }
$$

In terms of $\Lambda$ and $\phi$ :

$$
H_{0}=2 \mu\left[M^{2}\left(1-\Lambda^{2}\right)(1+\cos \phi)+\Lambda\right]=2 \mu H \text {. }
$$

Using standard, half-angle trignometric identities in Eq. (2.9) we obtain:

$H=\frac{2 M^{2}\left(1-\Lambda^{2}\right)}{1+\tau^{2}}+\Lambda$,

as a re-normalized form of the Hamiltonian $H$. Hamilton's Eqs. (2.1)-(2.2) are equivalent the Hamiltonian system $\phi_{\bar{x}}=$ $H_{\phi}$ and $w_{\bar{x}}=-H_{\phi}$ where $\bar{x}=2 \mu x$. From Eq. (2.2) we find:

$\frac{d \Lambda}{d x}=-\frac{M^{2} \sin \phi\left(1-\Lambda^{2}\right)}{\Lambda} \equiv-\frac{2 M^{2}\left(1-\Lambda^{2}\right) \tau}{\Lambda\left(1+\tau^{2}\right)}$.

Equation (2.11) can be reduced to an ordinary differential equation, expressed solely in terms of $\Lambda$, by solving the Hamiltonian integral (Eq. 2.10) for $\tau$ in terms of $\Lambda$ :

$\tau= \pm\left(\frac{2 M^{2}\left(1-\Lambda^{2}\right)-(H-\Lambda)}{H-\Lambda}\right)^{1 / 2}$.

Using Eq. (2.12) in Eq. (2.11) we obtain the differential equation:

$\frac{d \Lambda}{d x}=\mp \frac{\left\{(H-\Lambda)\left[2 M^{2}\left(1-\Lambda^{2}\right)-(H-\Lambda)\right]\right\}^{1 / 2}}{\Lambda}$,

for $\Lambda$. Equation (2.13) can be formally integrated to give a second integral for the system, namely:

$\int \frac{\Lambda d \Lambda}{\left\{(H-\Lambda)\left[2 M^{2}\left(1-\Lambda^{2}\right)-(H-\Lambda)\right]\right\}^{1 / 2}}=$

$-\sigma x+$ const.

where $\sigma=\operatorname{sgn}(\tau)=\operatorname{sgn}(\sin \phi)$.

It is also straightforward to obtain a similar differential equation for $\tau$ by using Eqs. (2.1) and (2.8) to first obtain the equation:

$\frac{d \tau}{d x}=2 M^{2}-\frac{1+\tau^{2}}{2 \Lambda}$.

Then solving the Hamiltonian integral (Eq. 2.10) for $\Lambda$ in terms of $\tau$, and substituting the result in Eq. (2.15) yields a differential equation for $\tau$ in which the right handside of Eq. (2.15) is solely a function of $\tau$. This differential equation is more complicated than the differential Eq. (2.13) for $\Lambda$ (note there are two solutions for $\Lambda$ as a function of $\tau$ obtained by solving Eq. 2.10).

The integral in Eq. (2.14) is an elliptic integral. The nature of the integral depends on the values of $M$ and $H$. Equation (2.13) can also be written in the potential well form:

$\left(\frac{d \Lambda}{d x}\right)^{2}=\frac{(H-\Lambda)\left[2 M^{2}\left(1-\Lambda^{2}\right)-(H-\Lambda)\right]}{\Lambda^{2}} \equiv$

$V(\Lambda)$.

For real solutions of Eq. (2.16) we require $V(\Lambda)>0$. Also note that for a physically relevant solution, Eq. (2.8) implies that $\Lambda$ must lie in the range $0 \leq \Lambda \leq 1$. 
We note for later reference, that $V(\Lambda)$ may be factorized in the form:

$V(\Lambda)=\frac{2 M^{2}\left(\Lambda-\Lambda_{-}\right)\left(\Lambda-\Lambda_{+}\right)(\Lambda-H)}{\Lambda^{2}}$,

where

$\Lambda_{ \pm}=\frac{1 \pm \Delta^{1 / 2}}{4 M^{2}}, \quad \Delta=16 M^{4}+1-8 M^{2} H$,

and $\Lambda_{ \pm}$are the roots of the quadratic factor in square braces in Eq. (2.16).

We could at this point proceed to consider the second integral (Eq. 2.14) in further detail. However, before proceeding, it is useful first to establish the nature of the Hamiltonian critical points.

\subsection{Hamiltonian critical points}

The Hamiltonian critical points occur at locations in the $(\phi, w)$ phase-plane, where

$H_{0 \phi}=H_{0 w}=0$.

At these points $\phi_{x}=w_{x}=0$. From Eqs. (2.1)-(2.2) the critical point conditions (Eq. 2.19) reduce to:

$$
\begin{aligned}
& H_{0 \phi}=-2 M^{2} w \sin \phi=0, \\
& H_{0 w}=2 M^{2}(1+\cos \phi)-(1-w / \mu)^{-1 / 2}=0 .
\end{aligned}
$$

There are two families of critical point solutions of Eqs. (2.20)-(2.21). The first family of critical points occur at the points $\left(\phi_{n 1}, w_{c 1}\right)$ :

$w_{c 1}=0, \quad \phi_{n 1}=2 n \pi \pm \phi_{c}$,

$\phi_{c}=\cos ^{-1}\left(\frac{1-2 M^{2}}{2 M^{2}}\right), \quad H=H_{c 1}=1$,

where $H_{c 1}$ is the value of the Hamiltonian at the critical points. Here $n$ is an integer, and it is assumed $M \geq 1 / 2$.

A second family of critical points occurs at the points $\left(\phi_{n 2}, w_{c 2}\right)$ :

$\phi_{n 2}=2 n \pi, \quad w_{c 2}=\frac{\mu\left(M^{4}-1 / 16\right)}{M^{4}}$,

$H_{c 2}=2 M^{2}+\frac{1}{8 M^{2}}$

where $H_{c 2}$ is the value of $H$ at the critical points, and $n$ is an integer. The solutions (Eq. 2.23) for $w=w_{c 2}$ are positive $\left(0<w_{c 2}<\mu\right)$ if $M \geq 1 / 2$. For solutions with $M \leq 1 / 2$, $w_{c 2}<0$ lies in the physically inadmissible region of the $(\phi, w)$ phase-plane.

Linearization of Hamilton's Eqs. (2.1)-(2.2) about the critical points yields the matrix system:

$$
\frac{d}{d x}\left(\begin{array}{c}
\delta \phi \\
\delta w
\end{array}\right)=\left(\begin{array}{cc}
H_{0 w \phi} & H_{0 w w} \\
-H_{0 \phi \phi} & -H_{0 \phi w}
\end{array}\right)\left(\begin{array}{c}
\delta \phi \\
\delta w
\end{array}\right) \equiv \mathbf{A}\left(\begin{array}{c}
\delta \phi \\
\delta w
\end{array}\right),
$$

where $\delta \phi=\phi-\phi_{c}$ and $\delta w=w-w_{c}$ denote the deviations of $\phi$ and $w$ from the critical point. Equations (2.24) have eigen-solutions of the form:

$\left(\begin{array}{c}\delta \phi \\ \delta w\end{array}\right)=\mathbf{r} \exp (\lambda x)$

where the eigenvector $\mathbf{r}=\left(r_{1}, r_{2}\right)^{T}$ satisfies the eigenvector equation:

$(\mathbf{A}-\lambda \mathbf{I}) \mathbf{r}=0$.

Equations (2.26) have non trivial solutions for $\mathbf{r}$ provided $\lambda$ satisfies the eigenvalue equation:

$\operatorname{det}(\mathbf{A}-\lambda \mathbf{I})=0$.

The solutions of Eq. (2.27) for $\lambda$ are:

$\lambda= \pm G^{1 / 2}$ where $G=-\operatorname{det} \mathbf{A}=H_{0 \phi w}^{2}-H_{0 \phi \phi} H_{0 w w}$.

Thus, the critical points are saddles if $G>0$ and are centers if $G<0$. It is interesting to note that the equation $\lambda^{2}=G$, i.e. $\lambda^{2}=H_{0 \phi w}^{2}-H_{0 \phi \phi} H_{0 w w}$ is the classical Monge-Ampere equation, which arises in the description of the Gaussian curvature of a 2D surface (e.g. Lipshutz, 1969; Von Mises, 1958; Ferapontov and Nutku 1994). Using the Hamiltonian Eq. (2.4) we find:

$G=M^{2}\left[4 M^{2} \sin ^{2} \phi-\frac{w}{\mu}\left(1-\frac{w}{\mu}\right)^{-3 / 2} \cos \phi\right]$,

at a general point $(\phi, w)$ in the phase plane.

Using Eqs. (2.26) and (2.29) we obtain the eigensolutions:

$\lambda_{1}=-\left(4 M^{2}-1\right)^{1 / 2}, \quad \mathbf{r}_{1}=(1,0)^{T}$,

$\left(\phi_{n 1}, w_{c 1}\right)=\left(2 n \pi+\phi_{c}, 0\right), \quad \lambda_{2}=\left(4 M^{2}-1\right)^{1 / 2}$,

$\mathbf{r}_{2}=\left(1,-4 \mu\left(4 M^{2}-1\right)^{1 / 2}\right)^{T}$,

for the eigenvalues and eigenvectors at the first family of critical points (Eqs. 2.22). For the first family of critical points we also have the solutions:

$\lambda_{1}=-\left(4 M^{2}-1\right)^{1 / 2}, \quad \mathbf{r}_{1}=\left(1,4 \mu\left(4 M^{2}-1\right)^{1 / 2}\right)^{T}$,

$\left(\phi_{n 1}, w_{c 1}\right)=\left(2 n \pi-\phi_{c}, 0\right)$,

$\lambda_{2}=\left(4 M^{2}-1\right)^{1 / 2}, \quad \mathbf{r}_{2}=(1,0)^{T}$,

Equations (2.30)-(2.31) show that the first family of critical points are saddles if $M>1 / 2$, and allow one to sketch the trajectories in the $(\phi, w)$ phase plane.

Similarly, from Eqs. (2.26)-(2.29) we obtain for the second family of critical points (Eq. 2.23) the eigensolutions:

$\lambda_{1}=-8 M^{2} i\left(M^{4}-1 / 16\right)^{1 / 2}$,
$\mathbf{r}_{1}=\left(1, \frac{i \mu\left(M^{4}-1 / 16\right)^{1 / 2}}{4 M^{4}}\right)$, 
Table 1. Critical points $\left(\phi_{n}, w_{n}\right)$ of the Hamiltonian (2.4) or (2.10) for the case $M \geq 1 / 2 . H_{c 2}=2 M^{2}+1 /\left(8 M^{2}\right)$ is the value of $H$ at the centre critical points.

\begin{tabular}{ccccc}
\hline$\phi_{n}$ & $w_{n}$ & Singularity Type & $\mathrm{H}$ & Comments \\
\hline & & & & \\
$2 n \pi \pm \phi_{c}$ & 0 & saddles & 1 & $\cos \phi_{c}=\left(1-2 M^{2}\right) /\left(2 M^{2}\right)$ \\
$2 n \pi$ & $\mu\left(M^{4}-1 / 16\right) / M^{4}$ & centres & $H_{c 2}$ & $H_{c 2} \geq 1$
\end{tabular}

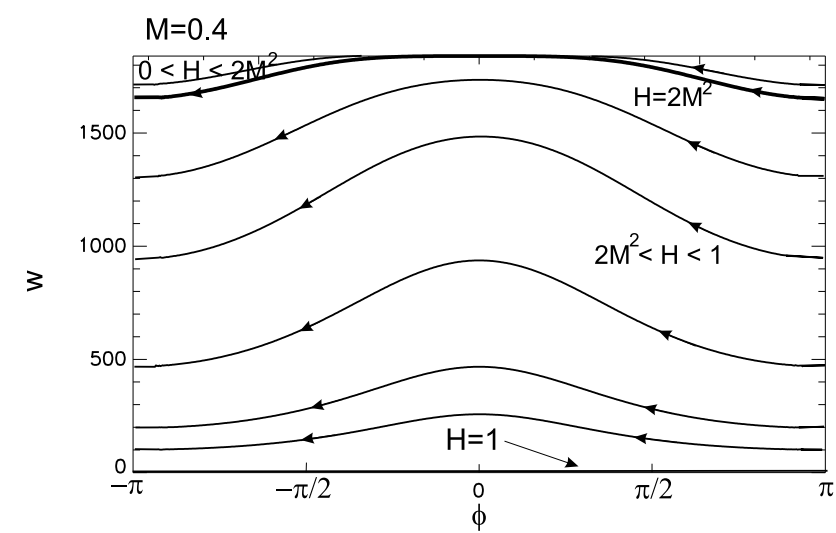

Fig. 3. Contours of the Hamiltonian $H$ in the $(\phi, w)$ phase plane for $0<M<1 / 2$. The arrows correspond to increasing $x$.

$\lambda_{2}=8 M^{2} i\left(M^{4}-1 / 16\right)^{1 / 2}$,

$\mathbf{r}_{2}=\left(1,-\frac{i \mu\left(M^{4}-1 / 16\right)^{1 / 2}}{4 M^{4}}\right)$.

The critical points are centres ( $O$-points) if $M>1 / 2$. If $M<1 / 2$, the critical points are saddles, but in that case $w=w_{c 2}<0$ lies in the unphysical region of the $(\phi, w)$ plane (i.e. $w_{c 2}<0$ ).

The basic properties of the two families of critical points (Eqs. 2.22 and 2.23) are summarized in Table 1.

\subsection{Phase plane trajectories}

The trajectories of the Hamiltonian system (Eqs. 2.1)-(2.4) using the canonical variables $(\phi, w) \equiv(q, p)$ can be written in the form:

$$
\begin{aligned}
& \frac{d \phi}{d x}=-\frac{\left(\Lambda^{2}-2 H \Lambda+1\right)}{\Lambda\left(1-\Lambda^{2}\right)} \\
& \frac{d w}{d x}=2 \mu \sigma\left\{(H-\Lambda)\left[2 M^{2}\left(1-\Lambda^{2}\right)-(H-\Lambda)\right]\right\}^{1 / 2}
\end{aligned}
$$

Hence the trajectories in the $(\phi, w)$ phase plane from Eqs. (2.34)-(2.35) are governed by the differential equation:

$$
\begin{aligned}
& \frac{d w}{d \phi}=-2 \mu \sigma \Lambda\left(1-\Lambda^{2}\right) \\
& \times \frac{\left\{(H-\Lambda)\left[2 M^{2}\left(1-\Lambda^{2}\right)-(H-\Lambda)\right]\right\}^{1 / 2}}{\Lambda^{2}-2 H \Lambda+1} .
\end{aligned}
$$

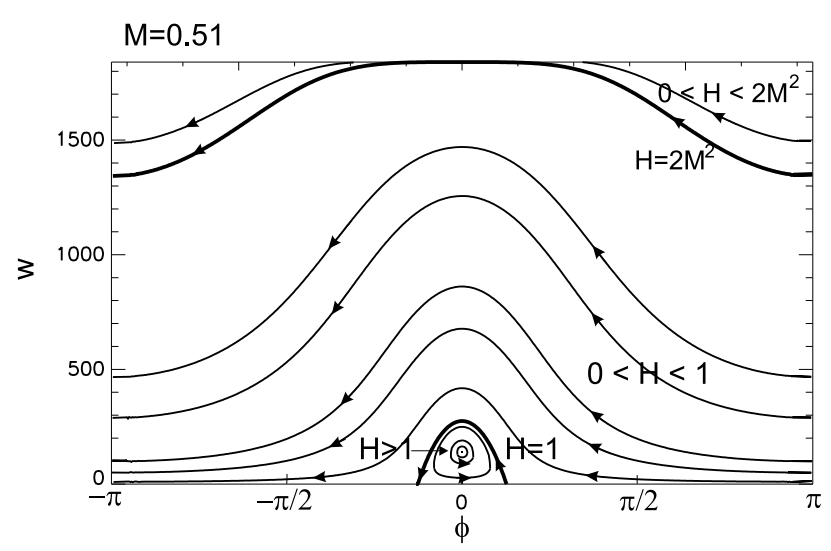

Fig. 4. Contours of $H$ in the $(\phi, w)$ phase plane for $1 / 2<M<$ $1 / \sqrt{2}$.

Equation (2.36) is equivalent to the equation $d w / d \phi=$ $-H_{\phi} / H_{w}$ where $H=$ const. is the Hamiltonian integral (Eqs. 2.10).

The importance of Eq. (2.36) is that it allows one to determine the values of $\Lambda$, and hence $w=\mu\left(1-\Lambda^{2}\right)$, in the phase plane where the phase trajectories are horizontal $(d w / d \phi=0)$ or vertical $(|d w / d \phi| \rightarrow \infty)$. Thus, for example, in order to obtain periodic solutions it is necessary for the phase trajectories to encircle the $O$-type critical points located at $\left(\phi_{n 2}, w_{c 2}\right)$ in Eq. (2.23). In order for this to be possible, it is necessary that the denominator:

$D(\Lambda)=\Lambda^{2}-2 H \Lambda+1$,

have real zeros for $\Lambda$ and that these zeros must lie in the physically allowed range $0 \leq \Lambda \leq 1$. From Eq. (2.37) $D(\Lambda)=0$ if

$\Lambda=\Lambda_{1,2}=H \pm \sqrt{H^{2}-1}$,

where the subscripts 1 and 2 are used to order the roots (i.e. $\Lambda_{1}<\Lambda_{2}$ ). From Eq. (2.38) a necessary condition for periodic solutions is that $H>1$.

Contours of the normalized Hamiltonian $H$ in the $(\phi, w)$ phase plane are illustrated in Figs. 3-6 for the cases: (a) $0<$ $M<1 / 2$, (b) $1 / 2<M<1 / \sqrt{2}$, (c) $M=1 / \sqrt{2}$ and (d) $M>1 / \sqrt{2}$, respectively. 


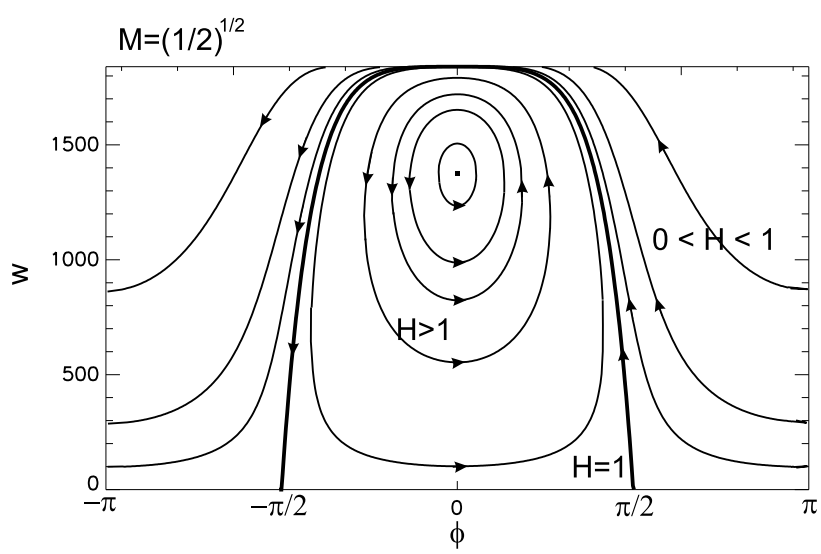

Fig. 5. Contours of $H$ in the $(\phi, w)$ phase plane for $M=1 / \sqrt{2}$.

Figure 3 shows phase-plane trajectories for the case $0<$ $M<1 / 2(M=0.4$ in the figure). In this case, there are no critical points for the Hamiltonian, and $0<H<1$ in the physically allowed region $(0<w<\mu)$. The phase trajectories split up into trajectories with $0<H<2 M^{2}$ which intersect the upper boundary at $w=\mu$ (i.e. $\Lambda=0$ ), where $d \phi / d x \rightarrow-\infty$, and a family of smooth solutions with $2 M^{2}<H<1$. The separatrix $H=2 M^{2}$ has an infinite derivative $d \phi / d x \rightarrow-\infty$ at $w=\mu(\Lambda=0)$, but $d w / d x=0$ at this point. The solutions with $2 M^{2} \leq H<1$ form solitary wave type structures with compact support (i.e. they have a finite spatial period, because the singularities of the integrand in Eq. (2.14) on the orbit are integrable). These solutions are analogous to compactons, which have been investigated in soliton theory (e.g. Rosenau and Hyman, 1993). One can concatenate a sequence of compactons to produce a modulated wave train, in which $u=\left|u_{e}\right|$ describes the wave envelope. Small amplitude, modulated wave trains of this type with $0<M<1 / 2$ were obtained by Dubinin et al. (2003) (it turns out that one can also obtain compactons for $1 / 2<M<1 / \sqrt{2}$ ). The phase trajectories in Fig. 3, show that one can obtain fully nonlinear compactons about a non-zero background state.

Figure 4 shows phase plane trajectories for a case with $1 / 2<M<1 / \sqrt{2}(\mathrm{M}=0.51)$. Closed periodic orbits occur if $1<H<H_{c 2}$ where $H_{c 2}=2 M^{2}+1 /\left(8 M^{2}\right)$ is the value of $H$ at the centre critical point. The $H=1$ contour corresponds to a solitary wave solution with an infinite spatial period and separates the periodic and non-periodic solutions. Only one period of the phase trajectories in $\phi$ are shown $(-\pi<\phi<\pi)$. Note that the contour $H=2 M^{2}$ is also a separatrix between the $0<H<2 M^{2}$ solutions which intersect the upper boundary $w=\mu$ (i.e. $\Lambda=0$ ) from the trajectories $2 M^{2}<H<1$ which do not intersect the boundary. Note $d w / d \phi=0$ at $w=\mu$ where $\Lambda=0$. ¿From Eq. (2.34) $d \phi / d x \rightarrow-\infty$ as $w \rightarrow \mu$ and $\Lambda \rightarrow 0$. At $\phi= \pm \pi, w=\mu\left(1-H^{2}\right)$ and $d w / d x=d w / d \phi=0$ for the $0<H<1$ contours. In assessing the phase plane trajec-

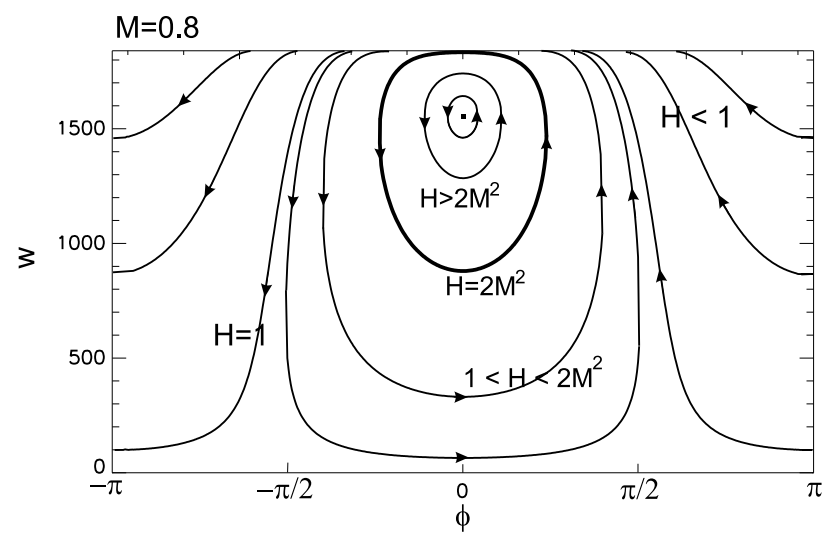

Fig. 6. Contours of $H$ in the $(\phi, w)$ phase plane for $M>1 / \sqrt{2}$. For periodic closed orbits requires $2 M^{2} \leq H<2 M^{2}+1 /\left(8 M^{2}\right)$.

tories it is useful to have at hand sketches of the function:

$Q(\Lambda)=(H-\Lambda)\left[2 M^{2}\left(1-\Lambda^{2}\right)-(H-\Lambda)\right]$

$=2 M^{2}\left(\Lambda-\Lambda_{-}\right)\left(\Lambda-\Lambda_{+}\right)(\Lambda-H)$,

versus $\Lambda$ showing the relative ordering of the zeros $\Lambda=\Lambda_{ \pm}$ and $\Lambda=H$, where $\Lambda_{ \pm}$are given by Eq. (2.18). From Eqs. (2.35)-(2.36) $d w / d x=d w / d \phi=0$ at these points (the corresponding values of $w$ are given by the formula $\left.w=\mu\left(1-\Lambda^{2}\right)\right)$. The trajectories with $2 M^{2}<H<1$ are compacton type solutions for which $w=u^{2} \rightarrow \mu\left(1-H^{2}\right)$ is finite as $\phi \rightarrow \pm \pi$ and $w \rightarrow \mu\left(1-\Lambda_{-}^{2}\right)$ at the center of the wave at $\phi=0$.

Figure 5 shows contours of $H$ for the case $M=1 / \sqrt{2}$. If one imagines continuously varying $M$ from $M<1 / \sqrt{2}$ up to $M=1 / \sqrt{2}$, then the separate contours $H=1$ and $H=2 M^{2}$ for $1 / 2<M<1 / \sqrt{2}$ merge in the limit as $M \rightarrow 1 / \sqrt{2}$. The solitary wave orbit $(H=1)$ now extends from the footpoints $[(\phi, w)=( \pm \pi / 2,0)]$ to the maximum allowed value of $w=\mu$ at $(\phi, w)=(0, \mu)$. At the maximum point, $d \phi / d x \rightarrow-\infty$ but $d w / d x=0$ where $\Lambda=0$ (see Eqs. 2.34-2.35). The periodic waves in this case correspond to contours with $1<H<H_{c 2}=1.25$. The contours with $0<H<1$ have the property that $d \phi / d x \rightarrow-\infty$ as $w \rightarrow \mu(\Lambda \rightarrow 0)$.

Figure 6 shows further evolution of the phase space trajectories (contours of $H$ ) for a case with $M>1 / \sqrt{2}(M=0.8)$. For $M>1 / \sqrt{2}$, the $H=1$ contour impinges on the upper boundary $w=\mu$ when $\tau=\tan (\phi / 2)= \pm\left(2 M^{2}-1\right)^{1 / 2}$ (see Eq. 2.12) and $\Lambda=0$. At this point $d w / d \phi=0$ but $d \phi / d x \rightarrow-\infty$. Hence it is not possible to obtain a solitary wave solution in this case, unless one inserts a shock jump somewhere before the trajectory hits the upper boundary (see Sect. 3 for further discussion of this point). There exists periodic travelling waves for $H$ contours in the range $1<2 M^{2}<H<H_{c 2}$ where $H_{c 2}=2 M^{2}+1 /\left(8 M^{2}\right)$. The $H=2 M^{2}$ contour is a periodic orbit in which $d \phi / d x \rightarrow$ $-\infty$ at $(\phi, w)=(0, \mu)$. However $d w / d x=0$ at this point. 


\subsection{Electron and proton phase variables $\phi_{e}$ and $\phi_{p}$}

From Eqs. (1.7) and (2.8) we obtain:

$\frac{d \tilde{\phi}}{d x}=\left(\frac{\mu-1}{\mu+1}\right) \frac{1}{\Lambda}$.

for the variation of $\tilde{\phi}=\phi_{e}+\phi_{p}$ throughout the wave. Alternatively using Eq. (2.13) we find:

$\frac{d \tilde{\phi}}{d \Lambda}=\left(\frac{\mu-1}{\mu+1}\right) \frac{1}{\Lambda d \Lambda / d x} \equiv-\frac{\sigma(\mu-1)}{(\mu+1) \sqrt{Q(\Lambda}}$,

where $Q(\Lambda)$ is given by Eq. (2.39) and $\sigma=\operatorname{sgn}[\tan (\phi / 2)]$. Thus, the solution for $\tilde{\phi}=\phi_{e}+\phi_{p}$ obtained by integrating Eq. (2.41) is:

$\tilde{\phi}=-\frac{\sigma(\mu-1)}{(\mu+1)} \int^{\Lambda} \frac{d \Lambda^{\prime}}{\sqrt{Q\left(\Lambda^{\prime}\right.}}$.

Since $\tilde{\phi}=\phi_{e}+\phi_{p}$ and $\phi=\phi_{p}-\phi_{e}$ the proton and electron phase variables $\phi_{p}$ and $\phi_{e}$ are given by:

$\phi_{p}=\frac{1}{2}(\phi+\tilde{\phi})$ and $\phi_{e}=\frac{1}{2}(\tilde{\phi}-\phi)$.

Equations (2.43) give the proton and the electron phase variables $\phi_{p}$ and $\phi_{e}$ in terms of $\phi$ and $\tilde{\phi}$. The solution for $\tilde{\phi}$ is given by Eq. (2.42) and the solution for $\phi$ is given by

$\phi=2 \tan ^{-1}(\tau)$,

where $\tau=\tau(\Lambda)$ is given by Eq. (2.12). The net upshot of the above analysis is that the solutions for electron and proton phase variables $\phi_{e}$ and $\phi_{p}$ are given parametrically in terms of $\Lambda$ by Eqs. (2.42)-(2.43). This then allows the determination of the complex transverse velocities $u_{e \pm}$ and $u_{p \pm}$ and the complex transverse magnetic fields $B_{ \pm}$throughout the wave.

\section{Solution examples}

From Eqs. (2.12) and (2.14) the travelling wave solution for $\tau=\tan (\phi / 2)$ and $\Lambda=\left(1-u^{2} / \mu\right)^{1 / 2}$ can be expressed in the parametric form:

$\tau=\sigma\left(\frac{2 M^{2}\left(1-\Lambda^{2}\right)-(H-\Lambda)}{H-\Lambda}\right)^{1 / 2}$,

$x=x_{0}-\sigma \int \frac{\Lambda d \Lambda}{\left\{(H-\Lambda)\left[2 M^{2}\left(1-\Lambda^{2}\right)-(H-\Lambda)\right]\right\}^{1 / 2}}$,

where $\sigma= \pm 1$ corresponds to the two solution branches, and $\sigma=\operatorname{sgn}(\tau)=\operatorname{sgn}(\sin \phi)$. It is implicitly assumed in Eqs. (3.1) and (3.2) that $\Lambda$ is restricted so that $x=x(\Lambda)$ and $\tau=\tau(\Lambda)$ are real.

The Hamiltonian critical point analysis in Eqs. (2.19)(2.33) shows that there are two families of critical points. The first family of critical points $\left(\phi_{n 1}, w_{c 1}\right)$ in Eq. (2.22) are saddles if $M>1 / 2$ and occur when $H=H_{c 1}=1$. The second family of critical points $\left(\phi_{n 2}, w_{c 2}\right)$ in Eq. (2.23) are centres if $M>1 / 2$, and correspond to $H=H_{c 2}=2 M^{2}+1 /\left(8 M^{2}\right)$. In this section, we first derive a solution connecting two saddle critical points at $(w, \phi)=\left(0, \phi_{c}\right)$ and $(w, \phi)=\left(0,-\phi_{c}\right)$, which is a heteroclinic orbit in the $(w, \phi)$ phase plane. This solution is a solitary wave solution for $u$ with an infinite spatial period in $x$, which has a bell shaped profile similar to that of the KdV soliton, and corresponds to the case where the Hamiltonian $H=1$. The oscilliton solutions investigated in this section correspond to the $H=1$ contours in the $(\phi, w)$ phase plane illustrated in Figs. 3-6.

Examples of both periodic and compacton wave packet solutions are given in Dubinin et al. (2003). However, the compacton type solutions were not identified as compactons as such (see Figs. 5 and 6 of Dubinin et al., 2003). We give further discussion of the compacton and periodic wave solutions in Sect. 4, where we determine the dependence of the wave period, $\mathrm{L}$, on $H$ and $M$.

\subsection{Case $H=1$}

From Eqs. (2.16)-(2.18), the integral (Eq. 3.2), for $H=1$, corresponds to the potential well equation:

$$
\left(\frac{d \Lambda}{d x}\right)^{2}=\frac{2 M^{2}(\Lambda-1)^{2}\left(\Lambda-\Lambda_{-}\right)}{\Lambda^{2}} \equiv V(\Lambda)
$$

where

$\Lambda_{-}=\frac{1-2 M^{2}}{2 M^{2}} \equiv \cos \phi_{c}, \quad \Lambda_{+}=1$,

are the roots of the quadratic equation:

$g(\Lambda)=-\left[2 M^{2}\left(\Lambda^{2}-1\right)+H-\Lambda\right]=0$,

where $g(\Lambda)$ is the quadratic factor appearing in Eqs. (3.1) and (3.2).

There are two distinct cases to consider, depending on the value of the Mach number $M$. For the case (a) $1 / 2<M<$ $1 / \sqrt{2}, 0<\Lambda_{-}<1$, whereas for the second case (b) with $M>1 / \sqrt{2},-1<\Lambda_{-}<0$. Sketches of the potential $V(\Lambda)$ in these two cases are given in Fig. 7. For a physical solution it is necessary that $V(\Lambda)>0$ and $0<\Lambda<1$.

¿From Eqs. (3.1)-(3.5):

$\tau=\sqrt{2} M \sigma\left(\Lambda_{-} \Lambda_{-}\right)^{1 / 2}$,

$x=x_{0}-\frac{\sigma}{\sqrt{2} M} \int^{\Lambda} \frac{\Lambda d \Lambda}{(1-\Lambda)\left(\Lambda-\Lambda_{-}\right)^{1 / 2}}$,

are the solutions for $\tau=\tau(\Lambda)$ and $x=x(\Lambda)$, where $x_{0}$ is an integration constant. We note for later reference, that the inverse of Eq. (3.6) giving $\Lambda$ as a function of $\tau$ is:

$\Lambda=\frac{1+\tau^{2}-2 M^{2}}{2 M^{2}}$.

Using $\tau$ to replace $\Lambda$ as integration variable in Eq. (3.7) we obtain:

$x=x_{0}+\frac{1}{M^{2}} \int^{\tau} \frac{1+\tau^{2}-2 M^{2}}{1+\tau^{2}-4 M^{2}} d \tau$, 
for the solution for $x$ in terms of $\tau$. The integral (Eq. 3.9) may be obtained in closed form:

$x=x_{0}+\frac{\tau}{M^{2}}+\frac{1}{\sqrt{4 M^{2}-1}} \ln \left|\frac{\tau-\sqrt{4 M^{2}-1}}{\tau+\sqrt{4 M^{2}-1}}\right|$.

Thus Eqs. (3.8)-(3.10) give an alternative parametric form for the solution in terms of $\tau$. Since $\Lambda=\left(1-u^{2} / \mu\right)^{1 / 2}$ we obtain:

$u^{2}=\mu\left(1-\Lambda^{2}\right) \equiv \frac{\mu}{4 M^{4}}\left[\left(4 M^{2}-1\right)-\tau^{2}\right]\left(1+\tau^{2}\right)$,

as the solution for $u^{2}$ in terms of $\tau$.

The phase variable $\tilde{\phi}=\phi_{e}+\phi_{p}$ in Eq. (2.42) may be expressed in the form:

$\tilde{\phi}=-\frac{\sigma(\mu-1)}{(\mu+1)} \int^{\Lambda} \frac{d \Lambda}{\sqrt{2} M(1-\Lambda)\left(\Lambda-\Lambda_{-}\right)^{1 / 2}}$.

Using Eq. (3.6), the integral (Eq. 3.12) reduces to:

$\tilde{\phi}=-\frac{2(\mu-1)}{(\mu+1)} \int^{\tau} \frac{d \tau^{\prime}}{\left(4 M^{2}-1\right)-\tau^{\prime 2}}=$

$\frac{(\mu-1)}{(\mu+1) \sqrt{4 M^{2}-1}} \ln \left|\frac{\tau-\sqrt{4 M^{2}-1}}{\tau+\sqrt{4 M^{2}-1}}\right|+\tilde{\phi}_{0}$.

The Eq. (3.13) is similar to that for $x$ in Eq. (3.10). Setting the integration constants $x_{0}$ and $\tilde{\phi}_{0}$ equal to zero in these equations, we obtain:

$\tilde{\phi}=\frac{\mu-1}{\mu+1}\left(x-\frac{\tau}{M^{2}}\right)$.

Hence from Eq. (2.43) the electron and proton phase variables $\phi_{e}$ and $\phi_{p}$ are given by:

$\phi_{e}=\frac{1}{2}\left[\frac{\mu-1}{\mu+1}\left(x-\frac{\tau}{M^{2}}\right)-\phi\right]$,

$\phi_{p}=\frac{1}{2}\left[\frac{\mu-1}{\mu+1}\left(x-\frac{\tau}{M^{2}}\right)+\phi\right]$.

The variables $\phi_{e}$ and $\phi_{p}$ govern the fine scale oscillations in the transverse electron and proton velocities $u_{e \pm}$ and $u_{p \pm}$ (see e.g. Fig. 2d).

$¿$ From Eqs. (1.8) and (1.9) the transverse magnetic field components $B_{y}$ and $B_{z}$ in the wave are given by:

$B_{y}=M_{A e}^{2} u\left(\cos \phi_{p}+\cos \phi_{e}\right) \equiv$

$2 M_{A e}^{2} u \cos \left(\frac{1}{2} \phi\right) \cos \left(\frac{1}{2} \tilde{\phi}\right)$,

$B_{z}=M_{A e}^{2} u\left(\sin \phi_{p}+\sin \phi_{e}\right) \equiv$

$2 M_{A e}^{2} u \sin \left(\frac{1}{2} \tilde{\phi}\right) \cos \left(\frac{1}{2} \phi\right)$.

Thus, the transverse magnetic field components exhibit wave beating between the electron and proton phases. From Eqs. (3.16)-(3.17)

$B_{\perp}=2 M_{A e}^{2} u \cos \left(\frac{1}{2} \phi\right)$,
a) $\quad \frac{1}{2}<M<\frac{1}{\sqrt{2}}$
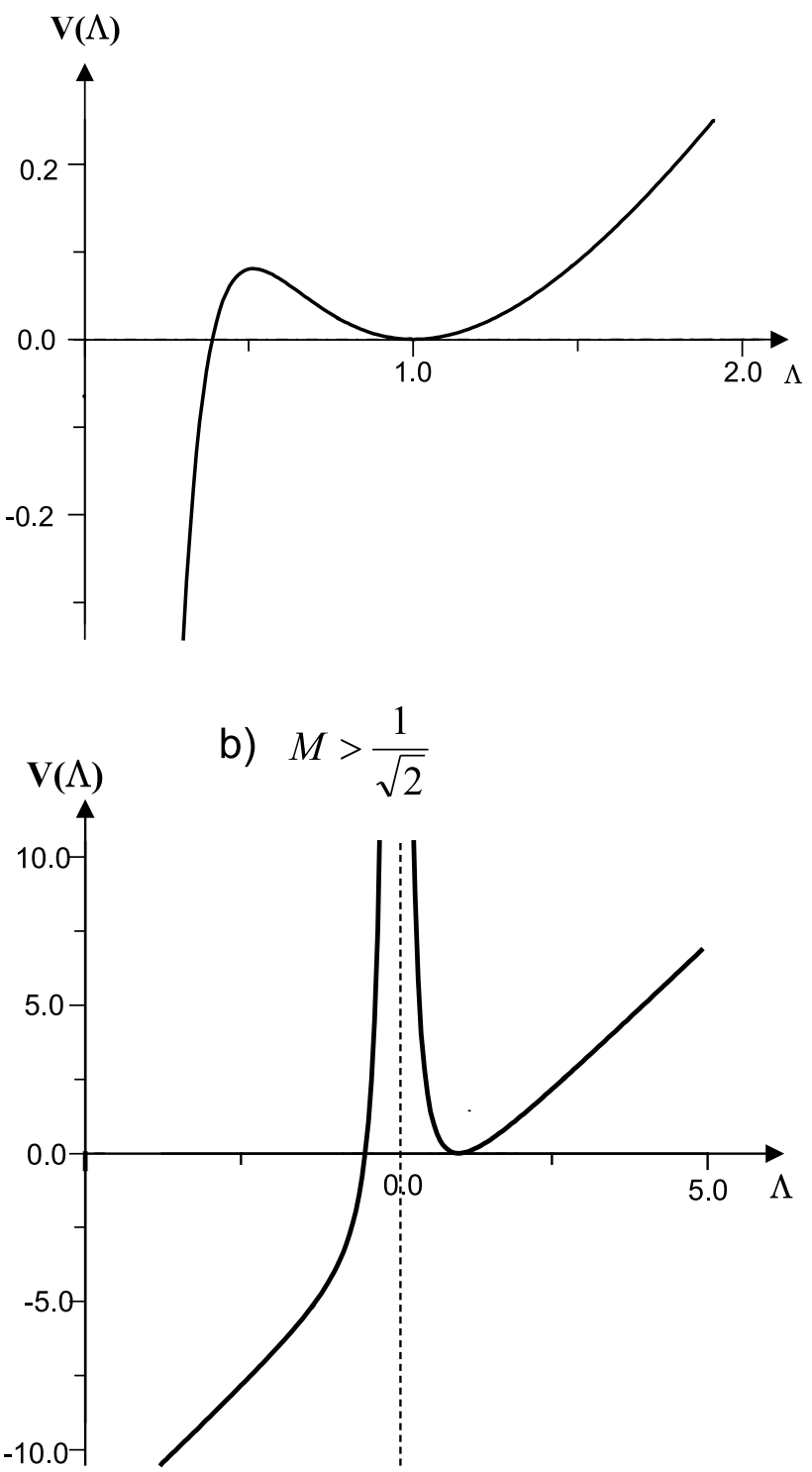

Fig. 7. Potential well diagrams of $V(\Lambda)$ versus $\Lambda$ from Eq. (3.3) for (a) $1 / 2<M<1 / \sqrt{2}$ and (b) $M>1 / \sqrt{2}$.

is the magnitude of the transverse field variations $\left(B_{\perp}=\sqrt{B_{y}^{2}+B_{z}^{2}}\right)$.

Below, we consider in more detail the large scale characteristics of the oscilliton travelling wave, with envelope governed by the parametric solutions $x=x(\tau)$ and $u=u(\tau)$ of Eqs. (3.10)-(3.11). Differentiating Eq. (3.9) implicitly with respect to $x$ we find $\tau$ satisfies the differential equation:

$\frac{d \tau}{d x}=\frac{M^{2}\left(1+\tau^{2}-4 M^{2}\right)}{1+\tau^{2}-2 M^{2}}$.

Equation (3.19) shows that $d \tau / d x=0$ at $\tau= \pm \tau_{c}$ where $\tau_{c}=\sqrt{4 M^{2}-1}=\tan \left(\phi_{c} / 2\right)$. From Eq. (3.11) $u=0$ at $\tau= \pm \tau_{c}$. The points $(u, \tau)=\left(0, \pm \tau_{c}\right)$ correspond 

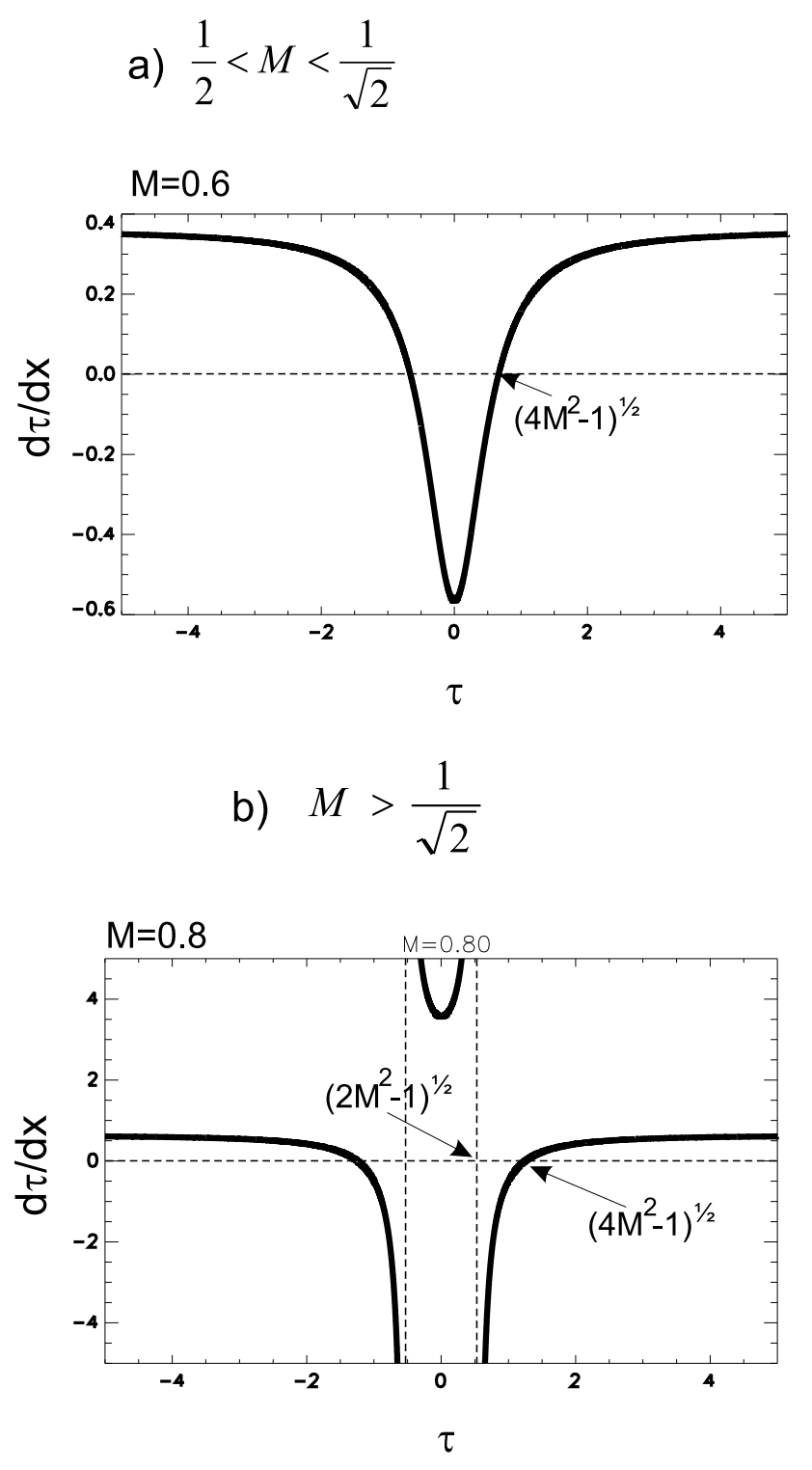

Fig. 8. Sketches of $d \tau / d x$ versus $\tau$ for the cases: (a) $1 / 2<M<$ $1 / \sqrt{2}$ and (b) $M>1 / \sqrt{2}$.

to the saddle critical points at $(w, \phi)=\left(0, \pm \phi_{c}\right)$ in the $(w, \phi)$ phase plane corresponding to the Hamiltonian contour $H=1$. Equation (3.19) also shows that $|d \tau / d x| \rightarrow \infty$ as $\tau \rightarrow \pm\left(2 M^{2}-1\right)^{1 / 2}$ if $M>1 / \sqrt{2}$.

For a physically relevant solution we require $0<\Lambda<1$. ¿From Eq. (3.8) this requires that $\tau$ can only have a limited range of values, namely:

(a) $|\tau|<\left(4 \mathrm{M}^{2}-1\right)^{1 / 2}$ if $1 / 2<\mathrm{M}<1 / \sqrt{2}$,

(b) $\left(2 \mathrm{M}^{2}-1\right)^{1 / 2}<|\tau|<\left(4 \mathrm{M}^{2}-1\right)^{1 / 2}$ if $\mathrm{M}>1 / \sqrt{2}$.

For $|\tau|<\left(2 M^{2}-1\right)^{1 / 2}$ in Case (b) $(M>1 / \sqrt{2})$, one obtains $\Lambda<0$, which is not allowed. The implications of the inequalities for the solutions can be seen, in part by sketching $\Lambda$ and $u^{2}$ as functions of $\tau$ for the two cases.
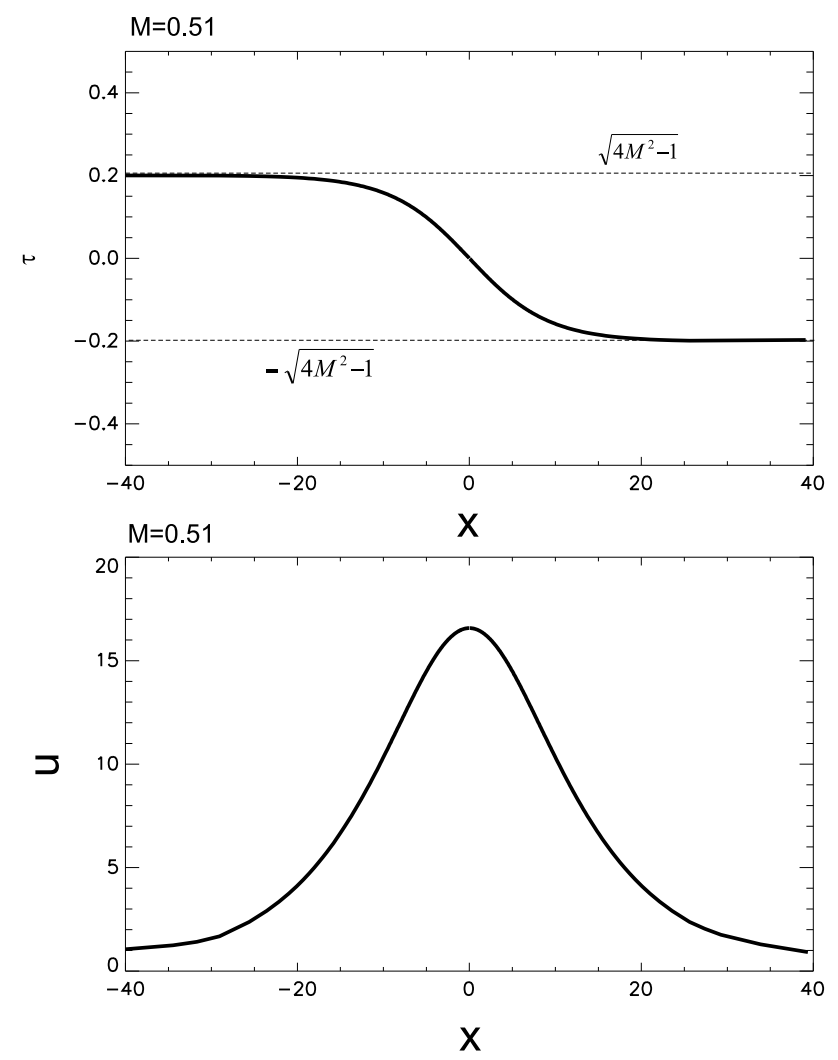

Fig. 9. Smooth travelling wave oscilliton profiles for $u$ and $\tau=$ $\tan (\phi / 2)$ for $1 / 2<M<1 / \sqrt{2}$ (Case (a)). The maximum of $u$ occurs at $x=0$ where $u=\sqrt{\mu} \sin \phi_{c}$ and $\sin \phi_{c}=\left(M^{2}-\right.$ $1 / 4)^{1 / 2} / M^{2}$.

For the case $M>1 / \sqrt{2}, \tau$ is not a monotonic, single valued function of $x$. This can be seen by sketching $d \tau / d x$ in Eq. (3.19) versus $\tau$ in the two cases (see Fig. 8). For Case (b), $d \tau / d x$ is negative if $\left(2 M^{2}-1\right)^{1 / 2}<|\tau|<\left(4 M^{2}-1\right)^{1 / 2}$, but is otherwise positive. Note that $d \tau / d x$ diverges as $|\tau| \rightarrow$ $\left(2 M^{2}-1\right)^{1 / 2}$ which indicates possibile shock formation, and multi-valued behaviour of $\tau$ as a function of $x$.

In Case (a), $d \tau / d x<0$ in the physically allowed region $|\tau|<\left(4 M^{2}-1\right)^{1 / 2}$. From Eq. (3.10), $x \rightarrow-\infty$ as $\tau \rightarrow$ $\left(4 M^{2}-1\right)^{1 / 2}$ and $x \rightarrow \infty$ as $\tau \rightarrow-\left(4 M^{2}-1\right)^{1 / 2}$. Also $x=0$ when $\tau=0$ if we choose the integration constant $x_{0}=0$. The solutions for $\tau(x)$ and $u(x)$ are illustrated in Fig. 9 for the case $M=0.51$. The solutions show that $\tau$ is a monotonic decreasing function of $x$, and the solution for $u(x)$ is reminiscent of the $\operatorname{sech}^{2}(x)$ profile characteristic of the KdV soliton.

In the limit as $M \rightarrow(1 / \sqrt{2})_{-}$, the profile for $d \tau / d x$ steepens, and has an infinite gradient at $x=0$. For example, for $M=1 / \sqrt{2}$, Eq. (3.19) reduces to:

$\frac{d \tau}{d x}=\frac{1}{2}\left(1-\frac{1}{\tau^{2}}\right)$, 

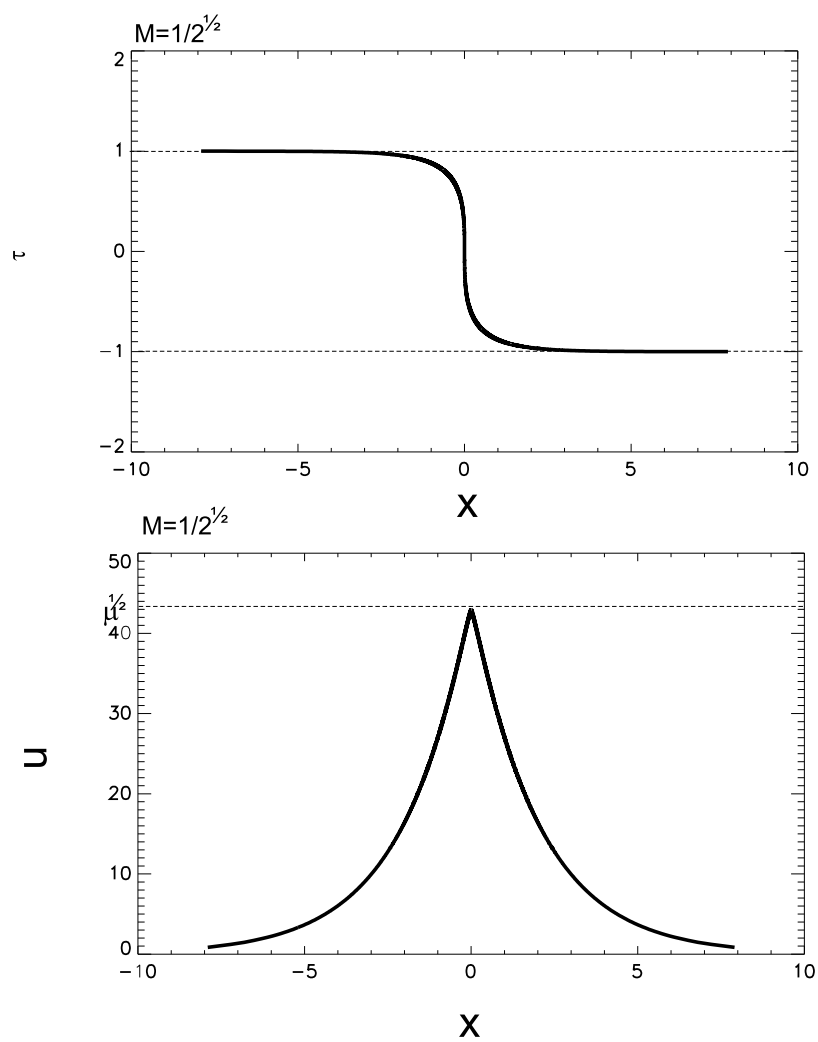

Fig. 10. Oscilliton solutions for $\tau(x)$ and $u(x)$ for $M=1 / \sqrt{2}$ and $H=1$.

and hence $d \tau / d x \rightarrow-\infty$ as $\tau \rightarrow 0$. The solution for $u^{2}$ reduces to:

$u^{2}=\mu\left(1-\tau^{2}\right)\left(1+\tau^{2}\right)$

Thus, the solutions for both $u$ and $\tau$ remain single valued as $M \rightarrow 1 / \sqrt{2}$. The maximum value of $u$ for this solution is $u=\sqrt{\mu}$ which is obtained at $x=0$ (we assume $x_{0}=0$ ). The solution for $M=1 / \sqrt{2}$ is illustrated in Fig. 10 .

For $M>1 / \sqrt{2}$ there does not exist a single valued solution for $\tau(x)$ stretching from $x=-\infty$ to $x=+\infty$. A solution profile (Eq. 3.11) for $\tau(x)$ for a case with $M>1 / \sqrt{2}$ $(M=0.9)$ is illustrated in Fig. 11. In the non-physical region $|\tau|<\left(2 M^{2}-1\right)^{1 / 2}, d \tau / d x>0$ and $d \tau / d x$ diverges as $\tau \rightarrow \pm\left(2 M^{2}-1\right)^{1 / 2}$. The solution could possibly be made single valued by inserting a shock in the flow. In the allowed physical region $\left(2 M^{2}-1\right)^{1 / 2}<|\tau|<\left(4 M^{2}-1\right)^{1 / 2}$ (see Eq. 3.20), $\tau(x)$ is a monotonic decreasing function of $x$. The nature of the shock could be complicated by the possibility of plasma heating, which would vitiate the cold plasma assumption used in the model.

The points $\mathrm{A}, \mathrm{B}$ and $\mathrm{C}$ on the solution profile $\tau(x)$ in Fig. 11 correspond to $\tau=\left(4 M^{2}-1\right)_{-}^{1 / 2},\left(4 M^{2}-2\right)^{1 / 2}$ and $\left(2 M^{2}-1\right)^{1 / 2}$ respectively. Similarly, D, E and F correspond to $\tau=-\left(2 M^{2}-1\right)^{1 / 2}, \tau=-\left(4 M^{2}-2\right)^{1 / 2}$ and $\tau=-\left(4 M^{2}-1\right)_{+}^{1 / 2}$ respectively. At points $\mathrm{B}, \mathrm{O}$ and $\mathrm{E}$, $u=\sqrt{\mu} \sin \phi_{c}$, and at $\mathrm{C}$ and $\mathrm{D}, u=\sqrt{\mu}$. One possible

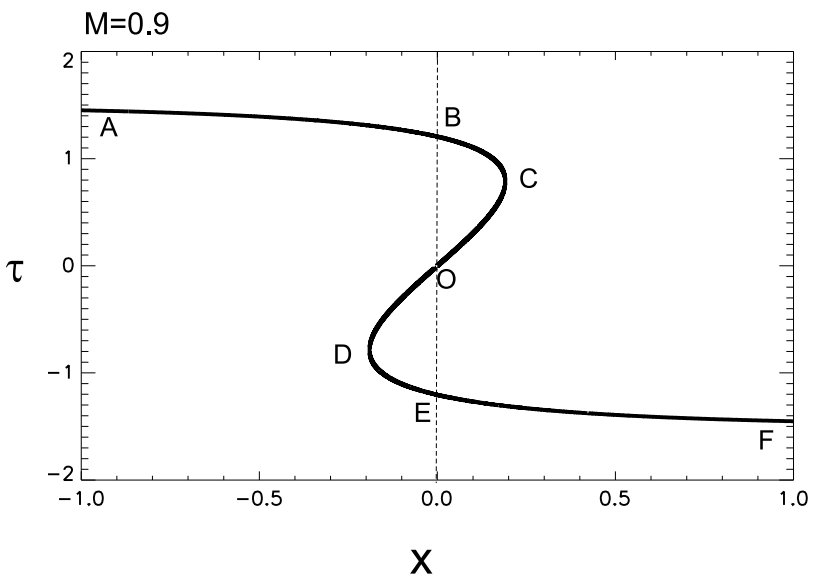

Fig. 11. Solution (Eq. 3.10) for $\tau(x)$ for $H=1$ and $M>1 / \sqrt{2}$. The solution becomes triple valued in the region near the origin about $x=0$.

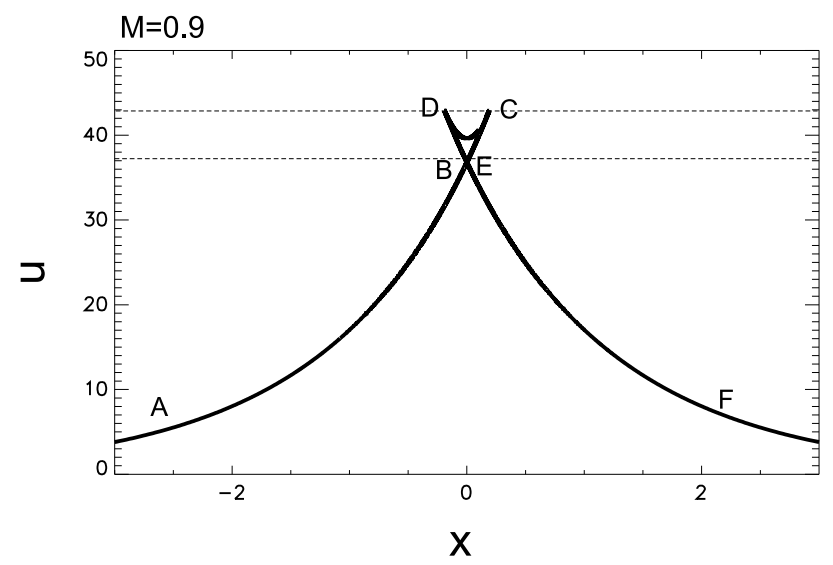

Fig. 12. Solution profile ABEF (solid line) for $u(x)$ for the rotational discontinuity shock solution of Fig. 11. Note B, E and O are the same point on the $u$ profile.

solution with a "shock jump" in $\phi$ consists of the profile $A B E F$ in Fig. 11 where there is a jump in $\phi$ at $x=0$. For this jump, there is no change in $u$ across the jump, since $u=\sqrt{\mu} \sin \phi_{c}$ at B,O and E. Effectively the transverse velocity $\mathbf{u}_{\perp}=u_{y} \mathbf{e}_{y}+u_{z} \mathbf{e}_{z}$ undergoes a rotation of $-2 \tan ^{-1}\left[\left(4 M^{2}-2\right)^{1 / 2}\right]$ through the transition. This proposed solution is more akin to a rotational discontinuity than a shock. The solution for $u(x)$ for this jump transition is illustrated in Fig. 12. The solution for $u(x)$ is given by the curve $A B E F$.

The proposed rotational discontinuity solution in Figs. 11 and 12 , implies that there must be a compensating rotation in the proton transverse velocity in order to ensure transverse momentum balance. Note that there is a discontinuity in $d u / d x$ at the jump, and that the maximum velocity $u_{\max }=\sqrt{\mu} \sin \phi_{c}$ is obtained at the discontinuity at $x=0$. 


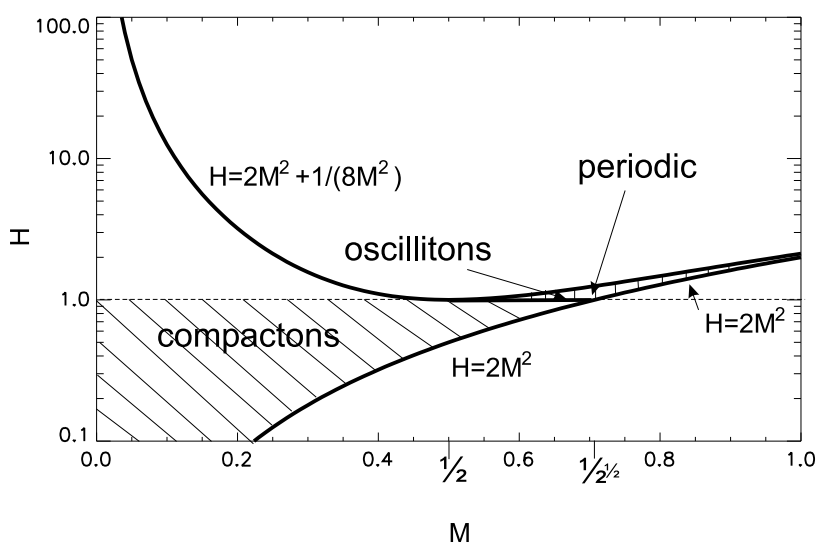

Fig. 13. Schematic of the $(M, H)$ plane indicating the regions where smooth compactons, oscillitons and periodic waves are obtained.

\section{Elliptic integrals and spatial periods}

In this section we discuss the nature of the solutions $x=$ $x(\Lambda)$ of the elliptic integral (Eq. 3.2) for $x$. The inverse of the elliptic integral gives the solution for $u^{2}=\mu\left(1-\Lambda^{2}\right)$ as a function of $x$. We show, that the elliptic integral can be split up into a linear combination of elliptic integrals of the first and second kind (Abramowitz and Stegun (1965), Ch. 17). We illustrate the use of these results by deriving some of the properties of the compacton solutions obtained in the parameter regime: $2 M^{2}<H<1$ and $0<M<1 / \sqrt{2}$ (see the $(\phi, w)$ phase plane trajectories in Figs. 3 and 4), and the periodic solutions obtained in the parameter regime $\max \left\{1,2 M^{2}\right\}<H<2 M^{2}+1 /\left(8 M^{2}\right)$ and $M>1 / 2$. A schematic of the $(M, H)$ plane in Fig. 13 illustrates the regions where one obtains compactons and periodic solutions. The oscillitons are seen to correspond to the limit of the compacton solutions as $H \uparrow 1$, and the limit of the periodic solutions in the limit as $H \downarrow 1$ in the Alfvén Mach number regime $1 / 2<M<1 / \sqrt{2}$. The oscillitons have an infinite spatial period, but the periodic and compacton solutions have finite periods depending on $H$ and $M$. We determine the dependence of the spatial period of the compactons and the periodic solutions, $L$, on the parameters $H$ and $M$.

\subsection{Compacton solutions}

¿From Eq. (3.2) the integral for $x=x(\Lambda)$ can be written in the form:

$x=x_{0}-\sigma \int^{\Lambda} \frac{\Lambda^{\prime}}{\sqrt{Q\left(\Lambda^{\prime}\right)}} d \Lambda^{\prime}$,

where

$Q(\Lambda)=(\Lambda-H)\left[2 M^{2} \Lambda^{2}-\Lambda+H-2 M^{2}\right] \equiv$

$2 M^{2}\left(\Lambda-\Lambda_{-}\right)\left(\Lambda-\Lambda_{+}\right)(\Lambda-H)$,

and $\Lambda_{ \pm}$are given by Eq. (2.18) (see also Eq. 2.39). For physical solutions we require that $Q(\Lambda)>0$ and that $0 \leq \Lambda \leq 1$.
In the compacton regime $\left(2 M^{2}<H<1\right.$ and $0<M<$ $1 / \sqrt{2}$ ), we find:

$0<\Lambda_{-}<H<1<\Lambda_{+}$.

Thus, $Q(\Lambda)>0$ if $\Lambda$ lies in the range: $\Lambda_{-}<\Lambda<H$. Hence in the compacton regime:

$x=-\sigma \int_{\Lambda_{-}}^{\Lambda} \frac{\Lambda^{\prime}}{\sqrt{Q\left(\Lambda^{\prime}\right)}} d \Lambda^{\prime}$,

is the form of the solution for $x=x(\Lambda)$, where we have set the constant of integration, $x_{0}$, equal to zero.

Introducing the new integration variable:

$y=\left(\frac{\Lambda^{\prime}-\Lambda_{-}}{H-\Lambda_{-}}\right)^{1 / 2}$,

the integral (Eq. 4.4) can be split up into a combination of elliptic integrals of the first and second kind, to obtain the solution for $x=x(\Lambda)$ in the form:

$x=-\frac{\sqrt{2} \sigma}{M\left(\Lambda_{+}-\Lambda_{-}\right)^{1 / 2}}\left[\Lambda_{+} U-\left(\Lambda_{+}-\Lambda_{-}\right) E(U \mid v)\right]$,

where

$$
\begin{aligned}
& U=\int_{0}^{\zeta} \frac{d y}{\sqrt{\left(1-y^{2}\right)\left(1-v y^{2}\right)}} \equiv \operatorname{sn}^{-1}(\zeta \mid v), \\
& E(U \mid v)=\int_{0}^{\zeta} d y\left(\frac{1-v y^{2}}{1-y^{2}}\right)^{1 / 2}, \\
& \zeta=\left(\frac{\Lambda-\Lambda_{-}}{H-\Lambda_{-}}\right)^{1 / 2} \equiv \operatorname{sn}(U \mid v), \quad v=\frac{H-\Lambda_{-}}{\Lambda_{+}-\Lambda_{-}},
\end{aligned}
$$

define standard elliptic integrals of the first and second kind respectively (Abramowitz and Stegun, 1965). Because of the ordering (Eq. 4.3), the parameter $v$ lies in the range: $0<v<1$. In Eq. (4.9), $\operatorname{sn}(U \mid v)$ is one of the standard Jacobian elliptic functions. The incomplete elliptic integral of the second kind (Eq. 4.8) can be expressed in a variety of different forms (e.g. by using the Jacobian Zeta function, which in turn can be expressed in terms of the Jacobian Theta function: Abramowitz and Stegun, 1965).

By noting that $\zeta=0$ when $\Lambda=\Lambda_{-}$and $\zeta=1$ when $\Lambda=H$, it follows from Eq. (4.6) that the spatial period of the compacton, $L$, is given by:

$$
\begin{aligned}
L & =\frac{2 \sqrt{2}}{M\left(\Lambda_{+}-\Lambda_{-}\right)^{1 / 2}}\left[\Lambda_{+} K-\left(\Lambda_{+}-\Lambda_{-}\right) E\right], \\
K & \equiv K(v)=\int_{0}^{1} \frac{d y}{\sqrt{\left(1-y^{2}\right)\left(1-v y^{2}\right)}} \\
E & \equiv E(K \mid v)=\int_{0}^{1} d y\left(\frac{1-v y^{2}}{1-y^{2}}\right)^{1 / 2},
\end{aligned}
$$

are complete elliptic integrals of the first and second kind. The result (Eq. 4.10) for the period $L$, corresponds to the change in $x$ moving along the compacton phase space trajectories from $\phi=\pi$ to $\phi=-\pi$ in Figs. 3 and 4 (note $\Lambda=H$ 
at $\phi= \pm \pi$ and $\Lambda=\Lambda_{-}$at $\phi=0$. In effect, Eqs. (4.10)(4.12) give the period of the compacton as a function of the two parameters $H$ and $M$ characterizing the solution.

As an example of the use of Eq. (4.10) for $0<M<1 / 2$, and for $H \sim 1$ (corresponding to the small amplitude compacton limit investigated by Dubinin et al., 2003), $\Lambda_{-} \approx 1$, $\Lambda_{+} \approx\left(1-2 M^{2}\right) /\left(2 M^{2}\right), v \approx 0, K \approx \pi / 2, E \approx \pi / 2$ (Abramowitz and Stegun (1965), formulae (17.3.11) and (17.3.12), p. 591), we obtain:

$L \sim \frac{2 \pi}{\sqrt{1-4 M^{2}}}$.

Thus, in the limit as $M \rightarrow 0, L \sim 2 \pi$, whereas as $M \rightarrow 1 / 2$, $L \rightarrow \infty$ (note that the limit as $M \rightarrow 1 / 2$ in this case is also the oscilliton solitary wave limit).

Similarly, for small $M$ we obtain:

$L \simeq \frac{2 \pi}{\left(1-4 M^{2} H\right)^{1 / 2}}\left[H-M^{2}(1-H)+O\left(M^{4}\right)\right]$.

Thus, in the limit as $M \rightarrow 0, L \simeq 2 \pi H$ if $H$ is $O(1)$.

From Eq. (2.42) the composite phase variable $\tilde{\phi}=\phi_{e}+\phi_{p}$ in the wave may be written as:

$\tilde{\phi}=-\frac{\sigma(\mu-1)}{(\mu+1)} \int_{\Lambda_{-}}^{\Lambda} \frac{d \Lambda^{\prime}}{\sqrt{Q\left(\Lambda^{\prime}\right)}}+\tilde{\phi}_{0}$.

Using the change of integration variable Eq. (4.5) we obtain:

$\tilde{\phi}-\tilde{\phi}_{0}=-\frac{\sqrt{2} \sigma(\mu-1)}{\left(\Lambda_{+}-\Lambda_{-}\right)^{1 / 2} M(\mu+1)} \operatorname{sn}^{-1}(\zeta \mid \nu)$,

where $\operatorname{sn}^{-1}(\zeta \mid v)=U$ is the function of $\Lambda$ defined in Eqs. (4.7)-(4.9). Equation (4.16) can be inverted to give:

$$
\begin{aligned}
& \Lambda=\Lambda_{-}+\left(H-\Lambda_{-}\right) \\
& \times \operatorname{sn}^{2}\left(\frac{\mu+1}{\mu-1} \frac{\left(\Lambda_{+}-\Lambda_{-}\right)^{1 / 2} M}{\sqrt{2}}\left(\tilde{\phi}-\tilde{\phi}_{0}\right) \mid \nu\right) .
\end{aligned}
$$

This latter formulation shows that the travelling wave solution can be written parametrically in terms of $\tilde{\phi}$. The variables $\phi_{e}$ and $\phi_{p}$ or $\phi$ and $\tilde{\phi}$ describe the fine scale oscillations inside the wave envelope.

\subsection{Periodic solutions}

The periodic solutions (see Figs. 4-6 and 13) are obtained in the regimes:

(a) $1<\mathrm{H}<\mathrm{H}_{\mathrm{c} 2}$ and $1 / 2<\mathrm{M}<1 / \sqrt{2}$,

(b) $2 \mathrm{M}^{2}<\mathrm{H}<\mathrm{H}_{\mathrm{c} 2}$ and $\mathrm{M}>1 / \sqrt{2}$.

For physical solutions, we require $Q(\Lambda)>0$ and $0 \leq \Lambda \leq 1$ (see Eq. 4.2 et seq.). Thus, for physical solutions:

$0 \leq \Lambda_{-}<\Lambda<\Lambda_{+}<1<H$.

For periodic solutions, $d \phi / d x \rightarrow 0$ as $\Lambda \rightarrow \Lambda_{1}$, where

$\Lambda_{1}=H-\left(H^{2}-1\right)^{1 / 2}$, and $\Lambda_{-}<\Lambda_{1}<\Lambda_{+}$. The proof that $\Lambda_{1}$ lies in this range can be proved using Eq. (4.18).

The solution for $x=x(\Lambda)$ is again given by Eq. (4.4), but for the periodic solutions, $\Lambda$ is restricted to the range (Eq. 4.19). Introducing the integration variable:

$y=\left(\frac{\Lambda^{\prime}-\Lambda_{-}}{\Lambda_{+}-\Lambda_{-}}\right)^{1 / 2}$,

the integral (Eq. 4.4) for $x=x(\Lambda)$ reduces to the sum of two elliptic integrals of the first and second kind, in the form:

$x=-\frac{\sqrt{2} \sigma}{M\left(H-\Lambda_{-}\right)^{1 / 2}}\left[H \tilde{U}-\left(H-\Lambda_{-}\right) E(\tilde{U} \mid \tilde{v})\right]$,

where

$\tilde{U}=\int_{0}^{\tilde{\zeta}} \frac{d y}{\sqrt{\left(1-y^{2}\right)\left(1-\tilde{v} y^{2}\right)}} \equiv \operatorname{sn}^{-1}(\tilde{\zeta} \mid \tilde{v})$,

$E(\tilde{U} \mid \tilde{v})=\int_{0}^{\tilde{\zeta}} d y\left(\frac{1-\tilde{v} y^{2}}{1-y^{2}}\right)^{1 / 2}$,

$\tilde{\zeta}=\left(\frac{\Lambda-\Lambda_{-}}{\Lambda_{+}-\Lambda_{-}}\right)^{1 / 2} \equiv \operatorname{sn}(\tilde{U} \mid \tilde{v}), \quad \tilde{v}=\frac{\Lambda_{+}-\Lambda_{-}}{H-\Lambda_{-}}$,

where $\tilde{U}$ is an elliptic integral of the first kind and $E(\tilde{U} \mid \tilde{v})$ is the corresponding elliptic integral of the second kind. The parameter $\tilde{v}$ lies in the range $0<\tilde{v}<1$ for the ordering (Eq. 4.18). Note that $\tilde{v}$ is the inverse of the parameter $v$ in Eq. (4.9) which was used in describing the compactons.

By noting that $\tilde{\zeta}=0$ when $\Lambda=\Lambda_{-}$and $\tilde{\zeta}=1$ when $\Lambda=\Lambda_{+}$, it follows from Eq. (4.22) that the spatial period of the wave, $L$, is given by:

$L=\frac{2 \sqrt{2}}{M \sqrt{H-\Lambda_{-}}}\left[H K(\tilde{v})-\left(H-\Lambda_{-}\right) E(K \mid \tilde{v})\right]$,

where $K(\tilde{v})$ and $E(K \mid \tilde{v})$ are the complete elliptic integrals of the first and second kind (c.f. Eqs. 4.11 and 4.12) with parameter $\tilde{v}$. The period $L$ corresponds to the change in $x$ in moving around the closed phase space orbits in Figs. 3-6, in an anti-clockwise fashion, starting at $(\phi, w)=\left(0_{-}, w_{-}\right)$at the top of the trajectory where $w_{-}=\mu\left(1-\Lambda_{-}^{2}\right)$, and ending at $(\phi, w)=\left(0_{+}, w_{-}\right)$.

Using Eq. (4.15) and the transformations (Eq. 4.21) the phase $\tilde{\phi}=\phi_{e}+\phi_{p}$ may be expressed in the form:

$\tilde{\phi}=-\left(\frac{\mu-1}{\mu+1}\right) \frac{\sqrt{2} \sigma}{M\left(H-\Lambda_{-}\right)^{1 / 2}} \mathrm{sn}^{-1}(\tilde{\zeta} \mid \tilde{\mathcal{v}})+\tilde{\phi}_{0}$,

where $\tilde{\zeta}$ and $\tilde{v}$ are given by Eq. (4.25). Again, $\phi$ and $\tilde{\phi}$ or $\phi_{p}$ and $\phi_{e}$ govern the fine scale oscillations inside the wave envelope.

4.3 The solution family $H=2 M^{2}$

In order to obtain some idea of how the period of the waves (both compactons and periodic waves) depends on $M$ it is convenient to consider the solution family with $H=2 M^{2}$. 
For this family, one obtains compactons if $0<M<1 / \sqrt{2}$, and periodic waves for $M>1 / \sqrt{2}$. These solutions have an infinite derivative for $\left|\phi_{x}\right|$ when $\Lambda=0$ (i.e. when $u=\mu$ at $x=0$ ), but are otherwise a regular well behaved solution family. From Eq. (2.18):

$\Lambda_{-}=0, \quad \Lambda_{+}=\frac{1}{2 M^{2}}, \quad H=2 M^{2}$,

for the solutions of interest. The curve $H=2 M^{2}$ is the lower limiting curve in the $(M, H)$ plane in Fig. 13 describing the compacton and periodic solution regimes.

\subsubsection{Compacton solutions}

The compacton solutions from Eqs. (4.6) and (2.12) are described by the equations:

$x=-\frac{\sigma}{M^{2}}[U-E(U \mid v)]$,

$\tau \equiv \tan (\phi / 2)=\sqrt{2} \sigma M\left(\frac{\Lambda\left(\Lambda-1 /\left(2 M^{2}\right)\right)}{\Lambda-2 M^{2}}\right)^{1 / 2}$,

where $\sigma=\operatorname{sgn}(\sin \phi)$ and

$v=4 M^{4} \quad$ and $\quad \zeta=\frac{\Lambda^{1 / 2}}{\sqrt{2} M}$,

are the paramters used in the elliptic integrals $U$ and $E(U \mid v)$ in Eqs. (4.7)-(4.8). From Eq. (4.10) the spatial period of the compactons, $\mathrm{L}$, is given by:

$L=\frac{2}{M^{2}}[K(v)-E(K \mid v)]$,

where $K(v)$ and $E(K \mid v)$ are the complete elliptic integrals (Eqs. 4.11-4.12). It is useful to note that $K(v)$ and $E(K \mid v)$ may be expressed in terms of Gauss's Hypergeometric function in the form:

$K(v)=\frac{\pi}{2} F(1 / 2,1 / 2 ; 1, v)$,

$E(K, v)=\frac{\pi}{2} F(-1 / 2,1 / 2 ; 1, v)$,

(Abramowitz and Stegun, 1965), we obtain:

$L \simeq 2 \pi M^{2}\left[1+(3 / 2) M^{4}+O\left(M^{8}\right)\right] \quad$ as $\quad M \rightarrow 0$,

for the spatial period of the compactons for small $M$. The result $L \simeq 2 \pi M^{2}$ can also be derived by setting $H=2 M^{2}$ in Eq. (4.14). In the limit as $M \uparrow 1 / \sqrt{2}$ and $v \rightarrow 1$, we obtain the equation:

$K(v) \simeq \frac{1}{2} \ln \left(\frac{16}{1-v}\right) \equiv \frac{1}{2} \ln \left(\frac{16}{1-4 M^{4}}\right)$,

$E(K \mid v) \simeq 1$.

The result (Eq. 4.34) for $K(v)$ can be determined from a formula for $F(a, b ; a+b, v)$ in Abramowitz and Stegun (1965) (formula 15.3.10, p. 559) with $a=b=1 / 2$ and $v \approx 1$.
Thus, the spatial period of the compactons, using Eq. (4.31) in this limit may be approximated by:

$L \simeq 4\left[\ln \left(\frac{2}{\sqrt{1 / 4-M^{4}}}\right)-1\right] \quad$ as $\quad M \uparrow 1 / \sqrt{2}$.

The Eqs. (4.33) and (4.35) show that $L \rightarrow 0$ in the limit as $M \rightarrow 0$ and $L \rightarrow \infty$ as $M \uparrow 1 / \sqrt{2}$. The period $L$, shows a logarithmic divergence as one approaches the oscilliton solution for $M=1 / \sqrt{2}$.

The compacton solutions are characterized by:

$u_{\text {max }}^{2}=\mu, \quad u_{\text {min }}^{2}=\mu\left(1-H^{2}\right) \equiv\left(1-4 M^{4}\right)$,

and hence

$\Delta u^{2}=u_{\max }^{2}-u_{\min }^{2}=4 \mu M^{4}$,

where $u_{\max }$ and $u_{\min }$ are the maximum and minimum values of $u$. Thus, the maximum change in $u^{2}$ is obtained in the oscilliton limit as $M \rightarrow 1 / \sqrt{2}$ and $\Delta u^{2} \rightarrow \mu$.

\subsubsection{Periodic solutions}

The implicit solution for the periodic waves for $H=2 M^{2}$ and $M>1 / \sqrt{2}$ is given by

$x=-2 \sigma[\tilde{U}-E(\tilde{u} \mid \tilde{v})]$,

$\tau \equiv \tan (\phi / 2)=\sqrt{2} \sigma M\left(\frac{\Lambda\left(\Lambda-1 /\left(2 M^{2}\right)\right)}{\Lambda-2 M^{2}}\right)^{1 / 2}$,

where $\sigma=\operatorname{sgn}(\sin \phi)$ and

$\tilde{v}=\frac{1}{4 M^{4}}$ and $\tilde{\zeta}=\sqrt{2} M \Lambda^{1 / 2}$,

are the values of the $\tilde{v}$ and $\tilde{\zeta}$ to be used in the elliptic integrals (Eqs. 4.23 and 4.24). From Eq. (4.37) the spatial period is given by:

$L=4[K(\tilde{v})-E(K, \tilde{v})]$,

where $K(\tilde{v})$ and $E(K, \tilde{v})$ are the complete elliptic integrals (Eqs. 4.1 and 4.12) with parameter $\tilde{v}$.

In the limit as $M \downarrow 1 / \sqrt{2}$, Eq. (4.40) gives:

$L \simeq 4\left[\ln \left(\frac{2}{\sqrt{M^{4}-1 / 4}}\right)-1\right] \quad$ as $\quad M \downarrow 1 / \sqrt{2}$.

which is similar to the compacton period (Eq. 4.35) in the limit as $M \uparrow 1 / \sqrt{2}$. Thus, $L \rightarrow \infty$ in the oscilliton limit as $M \downarrow 1 / \sqrt{2}$. In the limit as $M \rightarrow \infty, \tilde{v} \rightarrow 0$ and we obtain from Eq. (4.40) the result:

$L \simeq \frac{\pi}{4 M^{4}}\left[1+3 /\left(32 M^{4}\right)+O\left(1 / M^{8}\right)\right]$,

in this limit. Thus, $L \rightarrow 0$ as $M \rightarrow \infty$.

For the periodic waves we find:

$u_{\max }^{2}=\mu, \quad u_{\min }^{2}=\mu\left(1-\frac{1}{4 M^{4}}\right), \quad \Delta u^{2}=\frac{\mu}{4 M^{4}}$. 
Equations (4.37) and (4.43) show that $\Delta u^{2}$ is an increasing function of $M$ for the compactons, but a decreasing function of $M$ for the periodic waves. The maximum value of $\Delta u^{2}$ is obtained in the oscilliton limit as $M \rightarrow 1 / \sqrt{2}$ and $\Delta u^{2} \rightarrow$ $\mu$.

The dependence of the period $L$ as a function of $M$ for both the compactons and the periodic waves for $H=2 M^{2}$ is illustrated in Fig. 14a. Figure 14b shows the corresponding change in $\Delta u^{2}$ of the waves, as a function of $M$. However, it is important to note that these results strictly only apply for the case $H=2 M^{2}$.

\section{Concluding remarks}

Sauer et al. (2002), Dubinin et al. (2003) and McKenzie et al. (2004) used the equations of two-fluid, electron proton plasmas to study a variety of nonlinear, travelling wave solutions of the equations, representing nonlinear stationary whistler and whistler oscilliton waves in the travelling wave frame. Dubinin et al. (2003) showed that for the case of parallel propagation, in which the constant background magnetic field $\mathbf{B}_{0}=B_{0} \mathbf{e}_{x}$ and the travelling wave velocity $\mathbf{U}$ are both along the $\mathrm{x}$-axis, that the equations could be reduced to two coupled differential equations for the transverse electron speed $u \equiv\left|u_{e}\right|$ and the phase difference $\phi=\phi_{p}-\phi_{e}$ characterizing the complex transverse velocities $u_{j \pm}=u_{j y} \pm i u_{j z}=u_{j} \exp \left( \pm i \phi_{j}\right), j=e, p$ of the electrons and the protons.

In this paper it was shown that the integral of the equations $\Phi=\Phi(\phi, u)=$ const . obtained by these authors, is in fact the Hamiltonian $H_{0}$ of the system (modulo a constant), in which $\phi$ and $w=u^{2}$ are the canonical variables. A second integral of the equations was also obtained in the form $x=I(\Lambda)$, where $I(\Lambda)$ is an elliptic integral, and $\Lambda=\left(1-u^{2} / \mu\right)^{1 / 2}\left(\mu=m_{p} / m_{e}\right.$ is the ratio of the proton and electron masses, and $u$ is the transverse electron speed measured in units of the electron Alfvén speed $V_{A e}$ ).

Hamilton's equations for the system: $\phi_{x}=H_{0 w}$ and $w_{x}=-H_{0 \phi}$ have two families of critical points provided $M \equiv M_{A} \geq 1 / 2\left(M_{A}\right.$ is the Alfvén Mach number (Eq. 1.4) based on the geometric mean mass of the protons and the electrons). One of the families consists of saddle points, and the other family consists of centres (O-points) (Sect. 2.1). The solution trajectories in the $(\phi, w)$ phase plane (Hamiltonian contours) reveal the existence of: (a) oscillitons (or solitary wave) type solutions corresponding to heteroclinic orbits connecting neighbouring saddles; (b) periodic solutions which encircle the $O$-points and (c) compacton type solutions corresponding to solitary wave solutions with compact support (i.e. with a finite wavelength and with $d u / d x=0$ at the endpoints of the wave). In addition, there exists other solutions, which intersect the boundary of the physically allowed region at $u=\sqrt{\mu}$, at which point $d \phi / d x \rightarrow-\infty$ (see Figs. 3-6).

A schematic of the $(M, H)$ parameter plane in Fig. 13 reveals that compacton solutions are obtained in the region

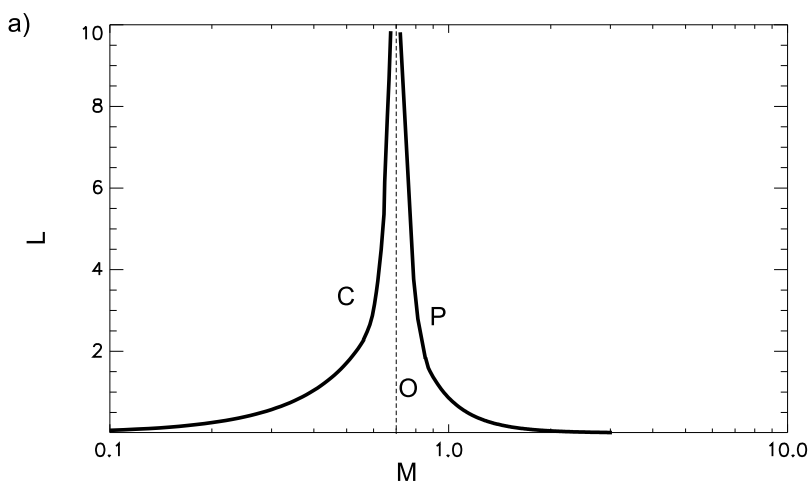

b)

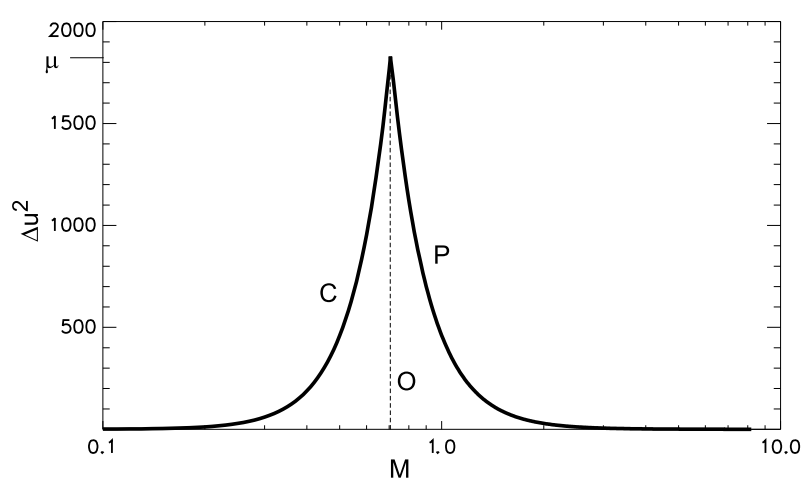

Fig. 14. Illustrates (a) the variation of the spatial period $L$ of the compactons $(C)$ and the periodic waves $(P)$ with the Alfvén Mach number $M$ for the solution family $H=2 M^{2}$, and (b) the variation of $\Delta u^{2}=u_{\max }^{2}-u_{\min }^{2}$ with $M$. The oscillitons $(O)$ are obtained in the limit as $M \rightarrow 1 / \sqrt{2}$.

$2 M^{2}<H<1$ and $0<M<1 / \sqrt{2}$; periodic solutions are obtained in the region $\max \left(1,2 M^{2}\right)<H<2 M^{2}+1 /\left(8 M^{2}\right)$ and $M>1 / 2$; and oscillitons are obtained on the common boundary $H=1$ and $1 / 2<M<1 / \sqrt{2}$ between the periodic and compacton regions. The detailed dependence of the spatial period $L$ of the compacton and periodic solution families on $H$ and $M$ are given in terms of complete elliptic integrals of the first and second kind (Eqs. 4.10 and 4.23). The period $L \rightarrow \infty$ as one approaches the oscilliton boundary region $H=1$ and $1 / 2<M<1 / \sqrt{2}$.

A detailed study of the oscilliton solutions with $H=1$ as $M$ varies from $1 / 2$ to $1 / \sqrt{2}$ was carried out (Figs. 7-11) using the analytical, implicit solution for $x$ and $\tau=\tan (\phi / 2)$ in terms of $\Lambda$ (Sect. 3). For $M>1 / \sqrt{2}$, the solution for $\tau=\tan (\phi / 2)$ becomes multiple valued near the origin. It was suggested that a solitary wave type solution, with an embedded rotational discontinuity, in which the transverse Reynolds stresses of the electrons are balanced by equal and opposite transverse stresses of the protons is possible in this case.

A Hamiltonian formulation using action-angle variables is developed in Appendix B. In this formulation, the action $I$ and angle variable $\theta$ are the canonical variables, and the Hamiltonian trajectories correspond to orbits on a torus. The action angle variables are also useful for determining the pe- 
riods of the periodic and compacton solutions. A further Hamiltonian formulation (Appendix C) uses $(\phi, \Lambda)$ as the canonical variables and $\tilde{\phi}=\phi_{p}+\phi_{e}$ as the new independent variable. In this development $x=x(\tilde{\phi})$ and the travelling wave solutions are expressed parametrically in terms of $\tilde{\phi}$.

We have used the terms "solitons" and "compactons" in this paper in a rather loose sense. In the mathematical literature (e.g. Rosenau and Hyman (1993)) solitons and compactons are solitary waves which undergo elastic collisions when two waves collide. A more accurate characterization of the travelling waves in this paper would be "solitary waves" and "compact solitary waves". Presumably in the small amplitude limit at least, one might expect the travelling waves to be solitons and compactons respectively. Spencer (1982), Spencer and Kaufman (1982), Holm and Kupershmidt (1983) and Sahraoui et al. (2003) have derived the general, Hamiltonian Poisson bracket structure of the multi-fluid plasma equations. This suggests that the Hamiltonian perspective will also provide further insight into the travelling wave oblique solitons in cold magnetized plasmas (McKenzie and Boyle, 2001), oblique oscillitons (Dubinin et al., 2003; McKenzie et al., 2004; McKenzie, 2004a; Verheest et al., 2004) and associated nonlinear, travelling waves in two-fluid plasmas (McKenzie et al., 2004a, b; McKenzie and Boyle, 2003; Sauer et al., 2001, 2002, 2003).

\section{Appendix A}

In this appendix we provide the basic equations for the travelling wave system (Eqs. 1.1-1.7) obtained by Dubinin et al. (2003). Dubinin et al. (2003) used the normalized variables:

$\bar{x}=\frac{X}{\ell_{e}}, \quad \ell_{e}=\frac{U}{\Omega_{e}}$,

where $X$ is the $x$-coordinate in the travelling wave frame. Dubinin et al. (2003) use the Alfvén Mach number $M_{A e}=$ $U / V_{A e}$ where $V_{A e}=B_{0} /\left(\mu_{0} n_{e} m_{e}\right)^{1 / 2}$ is the Alfvén speed based on the electron fluid. Note that

$\bar{x}=\alpha x, \quad M_{A e}^{2}=\alpha M_{A}^{2}, \quad \alpha=\frac{\mu+1}{\mu}$

relates the coordinates $\bar{x}$ and $x$ and $M_{A}^{2}$ to $M_{A e}^{2}$. The derivation of Eqs. (1.1)-(1.7) is based on Eqs. (A3)-(A8) given below, which follow from the work of Dubinin et al. (2003).

Using the above normalizations, Eqs. (1.1)-(1.2) are:

$$
\begin{aligned}
\frac{d u_{e}}{d \bar{x}} & =M_{A e}^{2} u_{e} \sin \phi, \\
\frac{d \phi}{d \bar{x}} & =2 M_{A e}^{2}(1+\cos \phi)-\alpha\left(1-u_{e}^{2} / \mu\right)^{-1 / 2},
\end{aligned}
$$

where

$\alpha=\frac{\mu+1}{\mu} \equiv \frac{m_{p}+m_{e}}{m_{p}}, \quad \mu=\frac{m_{p}}{m_{e}}$.

Equation (A4) slightly differs from the corresponding Eq. (30) of Dubinin et al. (2003) where $\alpha \rightarrow 1$. The correct version of Eq. (30) of Dubinin et al. (2003) is Eq. (A4).
The analogue of Eqs. (1.5)-(1.7) using the normalization (Eq. A1) are:

$$
\begin{aligned}
& \frac{d \phi_{e}}{d \bar{x}}=\frac{1}{\left(1-u_{e}^{2} / \mu\right)^{1 / 2}}-M_{A e}^{2}(1+\cos \phi), \\
& \frac{d \phi_{p}}{d \bar{x}}=M_{A e}^{2}(1+\cos \phi)-\frac{1}{\mu\left(1-u_{e}^{2} / \mu\right)^{1 / 2}}, \\
& \frac{d \tilde{\phi}}{d \bar{x}}=\frac{\mu-1}{\mu\left(1-u_{e}^{2} / \mu\right)^{1 / 2}}, \quad \tilde{\phi}=\phi_{p}+\phi_{e},
\end{aligned}
$$

The Eqs. (A6)-(A8) for $\phi_{e}, \phi_{p}$ and $\tilde{\phi}=\phi_{p}+\phi_{e}$ were not given explicitly in Dubinin et al. (2003), but are implicit in their analysis.

\section{Appendix B}

In this appendix we provide a discussion of the Hamiltonian system (Eqs. 2.1-2.2) in terms of action-angle variables. For "closed orbit" solutions, the Hamiltonian system (Eqs. 2.12.2) can be written in terms of action-angle variables $I$ and $\theta$ where

$$
\begin{aligned}
& I=\frac{1}{2 \pi} \oint p d q \equiv \frac{1}{2 \pi} \oint w\left(\phi, H_{0}\right) d \phi(x), \\
& S=\int^{\phi} w\left(\phi^{\prime}, H_{0}\right) d \phi^{\prime}, \quad \theta=\frac{\partial S(\phi, I)}{\partial I},
\end{aligned}
$$

where $I$ is the action (e.g. Zaslavsky et al., 1991). Note that $I=I\left(H_{0}\right)$ is a function of the Hamiltonian integral $H_{0}$. For the compacton solutions $-\pi \leq \phi<\pi$ corresponds to one period of the waves. For the periodic solutions, $-\phi_{1}<\phi<$ $\phi_{1}$ where $\left(-\phi_{1}, w_{1}\right)$ and $\left(\phi_{1}, w_{1}\right)$ are the points on the phase trajectories where $|d w / d \phi| \rightarrow \infty$. The sense of integration in Eq. (B1) is that of increasing $x$, which is indicated by the arrows on the phase space trajectories in Figs. 3-6.

Using Eqs. (2.1)-(2.2) and Eqs. (B1)-(B2) Hamilton's equations in terms of the action-angle variables, takes the form:

$\frac{d \theta}{d x}=\frac{\partial H_{0}}{\partial I}=\omega(I), \quad \frac{d I}{d x}=-\frac{\partial H_{0}}{\partial \theta}=0$,

which demonstrates that $(\theta, I)$ are canonically conjugate variables. In the derivation of Eq. (B3), $w=w\left(\phi, H_{0}\right)$ is regarded as a function of $\phi$ and $H_{0}$ and $I=I\left(H_{0}\right)$. Also note that

$\omega(I)=\frac{d H_{0}}{d I}, \quad \frac{\partial w\left(\phi, H_{0}\right)}{\partial H_{0}}=\frac{1}{\partial H_{0} / \partial w}=\frac{1}{\phi_{x}}$,

where use has been made of the Hamilton equation $\phi_{x}=$ $\partial H_{0} / \partial w$. The integrals of Eq. (B3) are:

$\theta=\omega(I) x+\theta_{0}, \quad I=I\left(H_{0}\right)=$ const..

Thus, in action-angle variables, the Hamiltonian trajectories lie on a torus. For periodic solutions, the trajectories form a closed curve on the torus. The spatial period of the wave is:

$L=\frac{2 \pi}{\omega(I)} \equiv 2 \pi \frac{d I}{d H_{0}}$. 
Using the expression (Eq. B1) for the action in Eq. (B6) we find:

$L=\oint \frac{\partial w\left(\phi^{\prime}, H_{0}\right)}{\partial H_{0}} d \phi^{\prime}(x) \equiv \oint \frac{1}{\partial H_{0} / \partial w} \phi_{x}^{\prime} d x \equiv \Delta x$,

where $\Delta x$ is the change in $x$ in moving around the orbit in the $(\phi, w)$ phase plane.

Using the results (Eqs. B1-B7, 4.1, and 4.2) we obtain:

$L=x\left(-\pi, w_{H}\right)-x\left(\pi, w_{H}\right)=2 \int_{\Lambda_{-}}^{H} \frac{\Lambda^{\prime}}{\sqrt{Q\left(\Lambda^{\prime}\right)}} d \Lambda^{\prime}$,

$I=-\frac{\mu}{\pi} \int_{\Lambda_{-}}^{H} \frac{\Lambda^{\prime 2}-2 \Lambda^{\prime} H+1}{\sqrt{Q\left(\Lambda^{\prime}\right)}} d \Lambda^{\prime}$.

for the period $L$ and action $I$ of the compactons, where $w_{H}=\mu\left(1-H^{2}\right)$ and $Q(\Lambda)$ is given by Eq. (4.2). The period $L$ in Eq. (B8) is equivalent to the result (Eq. 4.10). Similarly for the periodic waves, we obtain:

$L=x\left(0_{+}, w_{-}\right)-x\left(0_{-}, w_{-}\right)=2 \int_{\Lambda_{-}}^{\Lambda_{+}} \frac{\Lambda^{\prime}}{\sqrt{Q\left(\Lambda^{\prime}\right)}} d \Lambda^{\prime},(\mathrm{B} 10)$

$I=-\frac{\mu}{\pi} \int_{\Lambda_{-}}^{\Lambda_{+}} \frac{\Lambda^{\prime 2}-2 \Lambda^{\prime} H+1}{\sqrt{Q\left(\Lambda^{\prime}\right)}} d \Lambda^{\prime}$,

for the spatial period $L$ and the action $I$, where $w_{ \pm}=\mu(1-$ $\left.\Lambda_{+}^{2}\right)$. Note that $(\phi, w)=\left(0_{-}, w_{-}\right)$corresponds to the top of the orbit and $(\phi, w)=\left(0, w_{+}\right)$corresponds to the bottom of the orbit in the $(\phi, w)$-phase plane. The period (Eq. B10) is equivalent to Eq. (4.26).

\section{Appendix C}

In this appendix we discuss an alternative Hamiltonian formulation of the system (Eqs. 2.1-2.2) in which $\tilde{\phi}=\phi_{e}+\phi_{p}$ is used as the new independent variable to replace $x$. From Eq. (2.40) $x=x(\tilde{\phi})$ satisfies the differential equation:

$\frac{d x}{d \tilde{\phi}}=\frac{(\mu+1) \Lambda}{(\mu-1)}$

Noting that $w=\mu\left(1-\Lambda^{2}\right)$, the Hamiltonian system (Eqs. 2.1-2.2) can be cast in the alternative canonical form:

$\frac{d \phi}{d \tilde{\phi}}=\frac{\partial K}{\partial \Lambda}, \quad \frac{d \Lambda}{d \tilde{\phi}}=-\frac{\partial K}{\partial \phi}, \quad K=-\left(\frac{\mu+1}{\mu-1}\right) H$.

Thus, the Hamiltonian system (Eqs. 2.1-2.2) is equivalent to the Hamiltonian system (Eq. C2), with Hamiltonian $K$ and canonical variables $(\phi, \Lambda)$, with independent variable $\tilde{\phi}$. The transformation relating Eqs. (2.1)-(2.2) to Eq. (C2) is a type of canonical contact transformation (Goldstein, 1980). ¿From Eq. (C1),

$x=\left(\frac{\mu+1}{\mu-1}\right) \int_{0}^{\tilde{\phi}} \Lambda\left(\tilde{\phi}^{\prime}\right) d \tilde{\phi}^{\prime}+x_{0}$, where $x_{0}$ is an integration constant. The Hamiltonian system (Eq. C2) has the integral $H=$ const. (i.e. $K=$ const.) and the integral:

$\tilde{\phi}=-\sigma\left(\frac{\mu+1}{\mu-1}\right) \int_{\Lambda_{-}}^{\Lambda} \frac{d \Lambda^{\prime}}{\sqrt{Q\left(\Lambda^{\prime}\right)}}+\tilde{\phi}_{0}$,

where $\tilde{\phi}_{0}$ is an integration constant and $Q(\Lambda)$ is given by Eq. (4.2).

The above formulation, is particularly useful, since it allows one to write the oscilliton, compacton and periodic, travelling wave solutions parametrically in terms of $\tilde{\phi}$.

The oscilliton solution (Sect. 3.1) may be written parametrically in the form:

$\phi=2 \tan ^{-1}(\tau)=-2 \tan ^{-1}\left[\tau_{c} \tanh (k \tilde{\phi})\right]$,

$x=\left(\frac{\mu+1}{\mu-1}\right) \tilde{\phi}-\frac{\tau_{c}}{M^{2}} \tanh (k \tilde{\phi})$,

$u=\frac{\sqrt{\mu} \tau_{c}}{2 M^{2}} \operatorname{sech}(k \tilde{\phi})\left[1+\tau_{c}^{2} \tanh ^{2}(k \tilde{\phi})\right]^{1 / 2}$,

$\phi_{p}=\frac{1}{2}(\tilde{\phi}+\phi), \quad \phi_{e}=\frac{1}{2}(\tilde{\phi}-\phi)$,

$k=\frac{(\mu+1) \tau_{c}}{2(\mu-1)} . \quad \tau_{c}=\sqrt{4 M^{2}-1}$.

Note that $\tau_{c}=\tan \left(\phi_{c} / 2\right)$.

The compacton solution (Sect. 4.1) may be written parametrically in the form:

$\phi=-2 \tan ^{-1}\left[\Delta^{1 / 4} \operatorname{sc}(k \tilde{\phi} \mid v) d n(k \tilde{\phi} \mid \nu)\right]$,

$x=\frac{\mu+1}{\mu-1}\left[\Lambda_{+} \tilde{\phi}-\frac{\left(\Lambda_{+}-\Lambda_{-}\right)}{k} E(k \tilde{\phi} \mid \nu)\right]$,

$\Lambda=\Lambda_{-}+\left(H-\Lambda_{-}\right) \operatorname{sn}^{2}(k \tilde{\phi} \mid \nu), \quad u=\left[\mu\left(1-\Lambda^{2}\right)\right]^{1 / 2}$,

$\phi_{p}=\frac{1}{2}(\tilde{\phi}+\phi), \quad \phi_{e}=\frac{1}{2}(\tilde{\phi}-\phi)$.

Here

$\Lambda_{ \pm}=\frac{1 \pm \Delta^{1 / 2}}{4 M^{2}}, \quad \Delta=16 M^{4}+1-8 M^{2} H$,

$k=\left(\frac{\mu+1}{\mu-1}\right) \frac{\Delta^{1 / 4}}{2}, \quad v=\frac{H-\Lambda_{-}}{\Lambda_{+}-\Lambda_{-}}$,

$\operatorname{sc}(x \mid v), \operatorname{dn}(x \mid v), \operatorname{sn}(x \mid v)$ are Jacobian elliptic functions; $E(U \mid v)$ is a standard elliptic integral of the second kind. One period of the wave corresponds to $-K(v)<k \tilde{\phi}<K(v)$ where $K(v)$ is a complete elliptic integral of the first kind.

The periodic wave solutions (Sect. 4.2) can be written parametrically in terms of $\tilde{\phi}$ in the form:

$\phi=-2 \tan ^{-1}\left[\Delta^{1 / 4} \tilde{v}^{1 / 2} \operatorname{sn}(\tilde{k} \tilde{\phi} \mid \tilde{v}) \operatorname{cd}(\tilde{k} \tilde{\phi} \mid \tilde{v})\right]$,

$x=\left(\frac{\mu+1}{\mu-1}\right)\left[H \tilde{\phi}-\frac{\left(H-\Lambda_{-}\right)}{\tilde{k}} E(\tilde{k} \tilde{\phi} \mid \tilde{v})\right]$,

$\Lambda=\Lambda_{-}+\left(\Lambda_{+}-\Lambda_{-}\right) \operatorname{sn}^{2}(\tilde{k} \tilde{\phi} \mid \tilde{v}), \quad u=\left[\mu\left(1-\Lambda^{2}\right)\right]^{1 / 2}$,

$\phi_{p}=\frac{1}{2}(\tilde{\phi}+\phi), \quad \phi_{e}=\frac{1}{2}(\tilde{\phi}-\phi)$. 
In Eq. (C8)

$\tilde{v}=\frac{\Lambda_{+}-\Lambda_{-}}{H-\Lambda_{-}}, \quad \tilde{k}=\left(\frac{\mu+1}{\mu-1}\right) \frac{M\left(H-\Lambda_{-}\right)^{1 / 2}}{\sqrt{2}}$.

One period of the wave corresponds to the $\tilde{\phi}$ range: $0<$ $\tilde{k} \tilde{\phi}<2 K(\tilde{v})$.

Acknowledgements. The work of GMW is supported in part by NASA grant NAG5-13451 and NSF grant ATM-0317509. The work of E.D. is supported in part by Deutsche Forschungsgemeinschaft grant WO 910/1-1.

Edited by: J. Büchner

Reviewed by: one referee

\section{References}

Abramowitz, M. and Stegun, I. A.: Handbook of Mathematical Functions, (Dover: New York), 1965.

Dubinin, E., Sauer, K., and McKenzie, J. F.: Nonlinear stationary whistler waves and whistler solitons (oscillitons), Exact solutions, J. Plasma Phys., 69, 305-330, 2003.

Ferapontov, E. V. and Nutku, Y.: On the Monge-Ampere equivalent of the sine-Gordon equation, J. Phys. A, Math. and Gen., 27, 7831-7834, 1994.

Goldstein, H: Classical Mechanics, Addison Wesley, second edition, 1980.

Holm, D. D. and Kupershmidt, B. A.: Poisson brackets and Clebsch representations for magnetohydrodynamics, multi-fluid plasmas and elasticity, Physica D, 6, 347-363, 1983.

Lipshutz, M. M.: Theory and Problems of Differential Geometry, Schaum outline series, (McGraw Hill: NY), 1969.

McKenzie, J. F.: Some nonlinear stationary waves in space plasmas, Proc. 3rd Int. IGPP Conf., Physics of the outer heliosphere, edited by: Florinski, V., Pogorelov, N. V., and Zank, G. P., AIP Proc. Conf., 719, 287-292, 2004a.

McKenzie, J. F.: Nonlinear stationary waves of the ion-cylclotron type: solitons and spiky wave forms, J. Plasma Phys., 70, 533$541,2004 b$.
McKenzie, J. F. and Boyle, T. B.: Oblique solitons in a cold magnetized plasma, Physics of Plasmas, 8, 4367-4374, 2001.

McKenzie, J. F. and Boyle, T. B.: A unified view of acousticelectrostatic solitons in complex plasmas, New J. Phys., 5, 26.126.10, 2003.

McKenzie, J.F ., Dubinin, E., Sauer, K., and Doyle, T. B.: The application of the constants of the motion to nonlinear stationary waves in complex plasmas: a unified fluid dynamic viewpoint, J. Plasma Phys., 70, 431-462, 2004.

Rosenau, P. and Hyman, J. M.: Compactons: solitons with finite wavelength, Phys. Rev. Lett., 70, 5, 564-567, 1993.

Sahraoui, F., Belmont, G., and Rezeau, L.: Hamiltonian canonical formulation of Hall-magnetohydrodynamics: toward an application to weak turbulence theory, Physics of Plasmas, 10, 5, 13251337, 2003.

Sauer, K., Dubinin, E., and McKenzie, J. F.: New type of soliton in bi-ion plasmas and possible implications, Geophys. Res. Lett., 28, 3589-3592, 2001

Sauer, K., Dubinin, E., and McKenzie, J.F.: Coherent wave emission by whistler oscillitons: application to lion roars, Geophys Res. Lett., 29, 2226-2229, doi:10.1029/2002GL015771, 2002.

Sauer, K. Dubinin, E., and McKenzie, J. F.: Solitons and oscillitons in multi-ion plasmas, Nonlin. Processes Geophys., 10, 121-130, 2003 ,

\section{SRef-ID: 1607-7946/npg/2003-10-121}

Spencer, R. G.: The Hamiltonian structure of multi-species fluid electrodynamics, in: Mathematical methods in hydrodynamics and integrability in dynamical systems, edited by: Tabor, M. and Treve, Y. M., AIP Proc. Conf., 88, 121-126, 1982.

Spencer, R. G. and Kaufman, A. N.: Hamiltonian structure of twofluid plasma dynamics, Phys. Rev. A, 25, 4, 2437-2439, 1982.

Verheest, F., Cattaert, T., Dubinin, E., Sauer, K., and McKenzie, J. F.: Whistler oscillitons revisited: the role of charge neutrality?, Nonlin. Processes Geophys., 11, 447-452, 2004,

SRef-ID: 1607-7946/npg/2004-11-447.

Von Mises, R.: Mathematical theory of compressible fluid flows, Academic Press, New York, Art. 15.7-15.8, 231-233, 1958.

Zaslavsky, G. M., Sagdeev, R. Z., Usikov, D. A., and Chernikov, A. A.: Weak Chaos and Quasi-Regular Patterns, Cambridge University Press, Cambridge, UK, 1991. 